HEINZ MESSMER

\title{
Zur kommunikativen Neutralisierung sozialer Konflikte in den Verfahren des Rechts
}

\section{Recht und Sprache}

In Gerichtsverfahren wird typischerweise unter Anwesenden kommuniziert, und dies aus nahe liegenden Gründen. Mündlichkeit ist zunächst ein Gebot sämtlicher Prozessordnungen in den Verfahren des Rechts: Das Verwaltungsgericht entscheidet nach mündlicher Verhandlung ( 101 Abs. 1 VwGO), vor dem Zivilgericht verhandeln die Parteien des Privatrechtsstreits mündlich ( $\$ 128$ Abs. 1 ZPO) und auch der Strafrichter entscheidet nach der freien, aus dem Inbegriff der Verhandlung geschöpften Überzeugung ( $\$ 261 \mathrm{Abs}$. 1 StPO). Damit würdigt das Gericht den Grundsatz der Unmittelbarkeit eines persönlichen Eindrucks. Mündliche Verständigungsmodi sind zudem reaktionsschneller als die Schriftkommunikation und zur Entscheidung komplexer Problemstellungen besser geeignet. Zwischen Entstehung (was bereits geschehen ist) und Entscheidung eines Rechtsfalls (was noch nicht geschehen ist) hält Mündlichkeit das Nicht-Präsente im Bedarfsfall präsent und unter Entscheidungsgesichtspunkten flexibel verfügbar.? Mündlichkeit unterstützt damit die juristische Fallkonstitution, indem sie zwischen Sachverhaltsdarstellung und Rechtsnorm variabel changiert. ${ }^{2}$ Nicht zuletzt begründet das Mündlichkeitsprinzip elementare

${ }^{1}$ Zur Verknüpfung verschiedener Zeitebenen bei der rechtlichen Fallkonstitution vgl. WAYNE A. BEACH, Temporal Density in Courtroom Interaction: Constraints on the Recovery of Past Events in Legal Discourse, in: Communication Monographs 52 (1985), 1.

2 Nach LuDGER HOFFMANN, Vom Ereignis zum Fall. Sprachliche Muster zur Darstellung und Überprüfung von Sachverhalten vor Gericht, in: JÖRG SCHÖNERT (Hrsg.), Erzählte Kriminalität. Zur Typologie und Funktion von narrativen Darstellungen in Strafrechtspflege, Publizistik und Literatur zwischen 1770 und 1920. Tübingen 1991, 87, 89, bewegen sich in der juristischen Fallkonstitution "Sachverhaltsselektion und Konkretisierung des normativen Schemas (...) aufeinander zu, bis die Rechtsentscheidung möglich erscheint". Hoffmann zitiert in diesem Zusammenhang KARL ENGISCH, Logische Studien zur Gesetzanwendung. Heidelberg 1943, 15, der diesen Vorgang als "Hin- und Herwandern des Blicks" bezeichnet sowie THEODOR VIEHWEG, Topik und Jurisprudenz. 5. Aufl., München 1974, 90, der von der „wechselseitigen Annäherung zwischen Sachverhalt und Rechtsordnung" spricht. 
Aspekte der gerichtlichen Verfahrenslegitimität: Die „Darstellung eines Vorgangs der Entscheidungsfindung auf Grund von Normen" 3 ist eine wesentliche Bedingung für die Hinnahmebereitschaft der Entscheidungsbetroffenen auch dann, wenn gegen ihre Interessen entschieden wird.

Während Mündlichkeit die gerichtlichen Verfahrens- und Entscheidungsprozesse mit einer gewissen Elastizität ihrer Ablaufsstrukturen versorgt, sind ihr durch die Entscheidungszwecke selbst meist enge Grenzen gesetzt. Dies gilt insbesondere für das gerichtliche Strafverfahren. Kaum ein anderes Setting weist hinsichtlich Umfang und Inhalt von Äußerungen kommunikationsstrukturell vergleichbar rigide Einschränkungen auf. Mit dem Zwang, die Rechtsentscheidung auf eine im Grundsatz binär codierte Recht/Unrecht-Disjunktion ${ }^{4}$ zuzuführen, korrespondiert ein Verfahrensprozess, der zwischen relevanten und irrelevanten Informationen strikt unterscheidet. Der Zwang, den jeweiligen Sachverhalt mit der Rechtsnorm in Einklang zu bringen, bestimmt das Ausmaß kommunikativer Selektivität im Verfahren. Im Selbstverständnis der Rechtsprechung verknüpft das Gericht damit verschiedene Aufgaben der Sachverhaltsklärung, des Normbezugs und der Verfahrenslegitimität zu einem rational kalkulierbaren Entscheidungsprozess, dessen Gelingen von auffällig restriktiven Kommunikationsregeln abhängt.

Im kontinentaleuropäisch üblichen Gerichtsverfahren werden die prozessrechtlichen Kontrollbefugnisse durch den Richter ausgeübt. ${ }^{5}$ Richter steuern den Verfahrensablauf durch die Zuteilung von Rederechten und unterwerfen die Mitteilungsbedürfnisse und Äußerungs-

\footnotetext{
${ }^{3}$ Niklas Luhmann, Legitimation durch Verfahren. 3. Aufl., Darmstadt 1978, 107. Nach Luhmann ist die klassische Idee der Gerechtigkeit in der modernen Rechtsprechung insofern nicht mehr handlungsbegründend. Sie wird ersetzt durch die abstraktere - und aus systemtheoretischer Sicht anschlussfähigere - Problemlösungsformel adäquater Komplexität. Anstatt die Entscheidungsunterlegenen zu Konsens und Anerkennung zu motivieren, wird der Konsens über den Verfahrensausgang von diesen isoliert. Darauf kommen wir noch näher zu sprechen.

${ }^{4}$ Vgl. NikLas LuHManN, Das Recht der Gesellschaft. Frankfurt am Main 1993, 67 ff., der damit den Code und die daran anknüpfenden Voraussetzungen der operativen SchlieBung des Rechtssystems in der Gesellschaft bezeichnet.

${ }^{5}$ John Thibaut/Laurens WaLKer, Procedural Justice. Hillsdale (N. J.) 1975, 22 ff. unterscheiden in diesem Zusammenhang zwischen Parteiprozessmodell (adversary model) und Richtermodell (inquisitorial model). Im Unterschied zum Richtermodell wird im Parteiprozessmodell Art und Umfang der Beweisführung stärker von den Parteien bzw. von deren Prozessbevollmächtigten kontrolliert. Das hat einige Vorteile, allerdings auch den Nachteil, dass die Informationssammlung bzw. der Informationstransfer mitunter weniger effizient vonstatten geht als im vom Richter kontrollierten Verfahren.
} 
formen der Verfahrensbeteiligten dadurch ihrer Kontrolle. ${ }^{6}$ Geht es den Richtern vorwiegend um die Bewährung ihres Entscheidungsprogramms, so sind die Entscheidungsbetroffenen mehr am "Nettoeffekt ihrer Daseinsänderung " interessiert. ${ }^{7}$ Für die Entscheidungsbetroffenen führt dies mithin zu Problemen bei der Verteidigung ihrer Position. Auf Grund des selektiven Informationstransfers vor Gericht werden die übergreifenden Darstellungswünsche der Entscheidungsbetroffenen vielfach blockiert und gebrochen. Vor allem durch die Form der Befragung, die von den Betroffenen ein rasches Umschaltvermögen zwischen zusammenhängender Sachverhaltsschilderung und ihren speziellen Details bzw. zwischen innerer Einstellung und objektiver Wahrnehmung erfordert, werden die Gestaltungsmöglichkeiten ihrer Äußerungen hinsichtlich Inhalt, Umfang und Form stark beschnitten. ${ }^{8}$

Bis weit in die 80er Jahre hinein waren solche Strukturmuster der Gerichtskommunikation Anlass verschiedener sprach- und rechtstheoretischer Forschungen, häufig in system- und ideologiekritischer Absicht. Vor dem Hintergrund oppositionell eingestellter Gesellschaftsanalysen schrieb man dem Rechtssystem herrschafts- und bestandserhaltende Aufgaben zu, von denen man annahm, dass sie vorwiegend stratifikatorische, maßgeblich über soziale Schichtunterschiede laufende Differenzierungsmodi der Gesellschaft unterstützten. Fehlende Prozesskompetenzen, mangelnde Verfahrenstransparenz, gerichtliche Zeremonialität, Richterautorität und anderes mehr üben dieser Auffassung zufolge auf die Entscheidungsbetroffenen eine gezielt einschüchternde und lähmende Wirkung aus. Auf sprachlicher Ebene wurde einerseits der elaborierte Code der juristischen Rhetorik, andererseits der

\footnotetext{
${ }^{6}$ Vgl. näher RuDOLF WASSERMANN, Die richterliche Gewalt. Macht und Verantwortung des Richters in der modernen Gesellschaft. Heidelberg 1985, 193 ff.; DERS., Die Verminderung des Machtgefälles in der Strafgerichtsverhandlung als rechtspraktisches und rechtspolitisches Problem, in: Zeitschrift fiir Rechtspolitik 19 (1986), 133, $134 \mathrm{ff}$.

7 So eine treffende Formulierung von KARL F. SCHUMANN, Aushandeln von Sachverhalten innerhalb des Strafprozesses, in: HANS-GeORG SOEFFNER (Hrsg.), Interpretative Verfahren in den Text- und Sozialwissenschaften. Stuttgart 1979, 10, 11.

${ }^{8}$ Dazu näher WERNER KALLMEYER, Mündliche Kommunikation vor Gericht, in: RUDOLF WASSERMANN (Hrsg.), Recht und Sprache. Beiträge zu einer bürgerfreundlichen Justiz. Heidelberg 1983, 139, der zeigt, wie die richterlichen Fragetechniken die Befragten zu komplizierten Neuorientierungen ihrer jeweiligen Darstellungsfigur zwingen; ferner ELISABETH MÜLler-LuCKMANN, Die Psychologie der Befragung, in: RUdOlf WASSERMANN (Hrsg.), Menschen vor Gericht. Neuwied 1979, 69. Eines von vielen Beispielen in dieser Richtung erläutert RUTH WODAK, The Interaction Between Judge and Defendant, in: TEun A. VAN DifK (Hrsg.), Handbook of Discourse Analysis. Vol. 4: Discourse Analysis in Society. London 1985, 181, 187 f., innerhalb einer kurzen Interaktionssequenz zwischen Richter und (weiblicher) Beklagten (schwerer Verkehrsunfall), in der dieser die Darstellung der Beklagten mit geschlechtstypischen Einwänden mutwillig irritiert, beispielsweise mit der Frage, ob sie wisse, dass das Fahrzeug auch ein Lenkrad besitze.
} 
restringierte $\operatorname{Code}^{9}$ der Entscheidungsbetroffenen als Transmissionsriemen schichtmäßiger Ungleichheiten ausgemacht. Mit Blick auf den Gleichheitsgrundsatz der Rechtsprechung ließen sich daraus die entsprechenden Rückschlüsse ziehen. Kritisch beispielsweise wurde vermerkt, dass sich das richterliche Urteilsvermögen insbesondere für solche Informationen offen und zugänglich zeigt, die ihm verständlich und nachvollziehbar erschienen; unverstandene Sachverhalte dagegen würden in vertraute Denkkontexte umkontextiert und mithin als negative Eigenschaft der Person bzw. deren Verhalten zugerechnet. ${ }^{10}$ Vor diesem Hintergrund rückten auch soziale Degradierungs- und moralische Entrüstungszeremonien als richterliche Selbstimmunisierungstechniken vermehrt in den Blick. ${ }^{11}$

Andere Forschungen hatten sich demgegenüber mehr auf die narrativen Darstellungsmuster als Mittel der Glaubhaftmachung von Sachverhaltsdarstellungen konzentriert. Narrationen seien für die Glaubhaftmachung von Sachverhaltsdarstellungen besonders geeignet, sofern sie die Faktenvielfalt und Fallkomplexität von Ereigniszusammenhängen in ein konsistentes Informationsarrangement einbinden könnten. Erst dadurch werde der übergreifende Kontext von Tatsachenzusammenhängen für andere kognitiv und normativ sichtbar. ${ }^{12}$ Dementsprechend hingen die (quantitativ ohnehin geringen) Einfluss-

${ }^{9}$ Diese Unterscheidung stammt bekanntlich von BASIL BERNSTEIN, Studien zur sprachlichen Sozialisation. Düsseldorf 1972, 155 ff.; vgl. auch PAUL ATKINSON, Language, Structure and Reproduction: An Introduction to the Sociology of Basil Bernstein. London 1985.

${ }^{10}$ So etwa WASSERMANN, Die richterliche Gewalt (Fn. 6), 151, zum Problem richterlicher Introspektion. Gleicher bzw. ungleicher sozialer Hintergrund zwischen Richtern und Entscheidungsbetroffenen (bzw. Sozialprestige) wird somit zu einer wesentlichen Einflussvariable richterlicher Entscheidungspräferenzen, vgl. DONALD BLACK, Sociological Justice. New York 1989, VII; ferner STANTON WHEELER/KENNETH MANN/AuSTIN SARAT, Sitting in Judgement. The Sentencing of White-Collar Criminals. New Haven 1988 160, mit der Vermutung, dass White-Collar Criminals für ihre Vergehen vergleichsweise milder bestraft würden, da Richter mit ähnlichem sozialen Hintergrund die Sanktionsfolgen besser einschätzen könnten.

${ }^{11}$ Klassisch HAROLD GARFINKEL, Conditions of Successful Degradation Ceremonies, in: American Journal of Sociology 61 (1956), 420, demzufolge Degradierungszeremonien auf die rituelle Zerstörung beschuldigter Personen zielen.

12 JOHN L. COMAROFF/SIMON A. ROBERTS, The Invocation of Norms in Dispute Settlement: The Tswana Case, in: IAN HAMNETT (Hrsg.), Social Anthropology and Law. London 1977,86 , sprechen in diesem Zusammenhang von einem paradigm of argument als dem Versuch, "to convey a coherent picture of relevant events and actions in terms of one or more (implicit or explicit) normative referents", betonen also den normativen Bezug als Organisationsprinzip narrativer Sachverhaltsdarstellungen; siehe auch W. LANCE BeNNETT/MARTHA S. FELDMAN, Reconstructing Reality in the Courtroom. London 1981, 5 mit dem Hinweis: „Stories are systematic means of storing, bringing up to date, rearranging, comparing, testing and interpreting available information about social behavior", die sich um eine central action gruppierten (ebd., 9, 47 ff.). 
möglichkeiten der Entscheidungsbetroffenen darüber hinaus auch von der Durchsetzung vorgefasster Kommunikationspläne ab. Werde deren Konsistenz durch die gerichtlichen Fragetechniken kontinuierlich unterbrochen bzw. ihr Darstellungsfluss durch ständiges Nachfragen zermürbt, so führe dies selbst bei gerichtserfahrenen Statusgruppen zu Prozessen signifikant abnehmender Eloquenz, so dass sich die Chancen auf Anerkennung der eigenen Sachverhaltsschilderung dementsprechend vermindern. ${ }^{13}$

Entsprechend hat es immer wieder Versuche gegeben, die Restriktionen der rechtlichen Kommunikation zu umgehen. Kritisch wurde beispielsweise vermerkt, dass die binäre Entscheidungslogik des Rechts in Verbindung mit ihren sprachlichen Mustern die Betroffenen ihrer Konflikte enteigne ${ }^{14}$, indem sie diese in eine für sie fremde und undurchschaubare Verfahrensstruktur zwinge, die ihnen dann auch hinsichtlich ihres Verfahrensergebnisses unverständlich bliebe. Damit stand letzthin die Idee der Gerechtigkeit in den Verfahren des Rechts zur Disposition. In den frühen 70er und 80er Jahren haben sich vor diesem Erkenntnishintergrund verschiedene Gegenströmungen in Form des Alternative Dispute Settlement ${ }^{15}$ entwickelt, von denen in den letzten zwei Dekaden besonders Mediationsverfahren stark an Bedeutung gewannen. ${ }^{16}$ In ihnen wird der binäre Entscheidungszwang (Recht/Unrecht) partiell fallen gelassen und durch gezielt inszenierte Kompromissbildungsprozesse ersetzt, die sich mehr am Konfliktlösungspotential der Betroffenen orientieren.

\footnotetext{
${ }^{13}$ Methodologisch anspruchsvolle und inhaltlich aufschlussreiche, aber letzthin wenig rezipierte Untersuchungen zu diesem Thema wurden in den 80er Jahren vom BKA in Auftrag gegeben mit dem Ziel, die kommunikativen Fertigkeiten und Durchsetzungschancen von Polizeibeamten als Zeugen vor Gericht zu verbessern, vgl. näher $\mathrm{H}$ WALTER SCHMITZ, Tatgeschehen, Zeugen und Polizei. Wiesbaden 1978; JOACHIM KNUF, Polizeibeamte als Zeugen vor Gericht. Eine kommunikationswissenschaftliche Untersuchung. Wiesbaden 1982, 271, mit dem Befund, dass den Betroffenen durch das kommunikative Regelwerk vor Gericht die Möglichkeit einer sachlich kompetenten Sprechweise genommen werde, so dass mitunter der Eindruck entsteht, diese könnten den kommunikativen Standards vor Gericht nicht folgen oder zu keinen konsistenten Darstellungsweisen finden.

${ }^{14}$ So die einprägsame Formulierung des norwegischen Rechtstheoretikers NILS CHRISTIE, Conflict as Property, in: The British Journal of Criminology 17 (1977), $1 \mathrm{ff}$.

$15 \mathrm{Vgl}$. anstatt vieler RICHARD L. ABEL (Hrsg.), The Politics of Informal Justice. New York 1982.

${ }^{16}$ Vgl. HeInZ MESSMER/HANS-UWE OTTO (Hrsg.), Restorative Justice on Trial: Pitfalls and Potentials of Victim-Offender Mediation. Dordrecht 1992; ANNE DIETER/LEO MONTA. DA/ANNEDORE SCHULZE (Hrsg.), Gerechtigkeit im Konfliktmanagement und in der Mediation. Frankfurt am Main 2000; HeInZ MESSMER, Mediation, in: HANS-UwE OTTO/HANS THIERSCH (Hrsg.), Handbuch zur Sozialarbeit/Sozialpädagogik. Neuwied 2001, 1169; FRITJOF HAFT/KATHARINA VON SCHLIEFFEN (Hrsg.), Handbuch Mediation. München 2002.
} 
Aus Sicht der Rechtsprechung dagegen wurde mit Nachdruck auf die Notwendigkeit des formal-rechtlichen Entscheidungsfindungsmechanismus insistiert - unter Inkaufnahme der damit verbundenen Probleme: Man könne auf die Rigidität gerichtlicher Entscheidungsverfahren nicht verzichten, ohne zugleich die Gültigkeit der rechtlichen Norm zu unterminieren. Die Sichtweisen des Rechts und die der Entscheidungsbetroffenen seien nun einmal zwangsläufig inkongruent, sofern sich diese über verschiedenartige Entscheidungsprämissen begründen. Es sei zudem nicht Sache des Gerichts, den Entscheidungsbetroffenen die Relevanzkriterien der Rechtsprechung zu erläutern und noch viel weniger, sich deren Wahrnehmungsprämissen zu Eigen zu machen. Bei der Anwendung von Recht und beim Lernen von Recht handle es sich um zwei diskrepante Funktionen, die nicht gleichzeitig optimiert werden könnten. ${ }^{17}$ Diese Argumentation stützt sich im Kern auf die überlegene Effizienz systeminterner Entscheidungsvorgänge gegenüber den einfachen, nicht hinreichend komplexen und zudem an einseitigen Interessen orientierten Entscheidungspräferenzen der jeweiligen Klientel. Die Form des gerichtlichen Verfahrens verfolge außerdem den grundsätzlichen Zweck, die Entscheidungsbetroffenen von ihren Widerstandsmöglichkeiten $z u$ isolieren, um sich gegenüber enttäuschungsbedingten Protesten $z \mathfrak{u}$ immunisieren. Dieser Umstand schlage sich zwangsläufig auch im Stil der Rechtssprache nieder. Die Darstellung der, gerichtlichen Wahrheit' ist oft mehr eine Frage der Form als über Inhalte vermittelt. Die Sprachformen, derer sich die Rechtsprechung zur Feststellung, wahrer' Sachverhalte bedient, zielten auf materialrechtlich verwertbare Wahrheit:

„Diese Sprache stellt fest. Sie gibt sich klar und sicher; sie muss dies tun, sonst gelingt die Mitteilung von Wahrheit nicht. Diese Sprache ist aber auch zögernd und ängstlich, weil sie weiß, dass unformalisierte wirkliche Wahrheit mit dem Mittel des Strafprozessrechts schwer zu gewinnen ist. Diese Sprache häuft Argument auf Argument, um ihre Unsicherheit zu überwinden. Sie vermeidet alle Risiken stilistischer Art; individuelle Stilformen entfernen den Autor von der Wahrheit. Diese Sprache lässt sich auf nur undeutlich fassbare Gegenstände, Gefühle z. B., nicht ein, entfernt diese Gegenstände damit aus dem Strafrecht." 18

\footnotetext{
17 LUHMANN, Legitimation durch Verfahren (Fn. 3), 114. Ähnlich argumentiert Luhmann auch im Hinblick auf Rechtssicherheitserwägungen: „Ein System, das die Entscheidbarkeit aller aufgeworfenen Probleme garantieren muss, kann nicht zugleich auch die Richtigkeit der Entscheidungen kontrollieren", (ebd., 21). Unter Entscheidungsgesichtspunkten wird der systeminternen Verfahrensrationalität somit Vorrang eingeräumt gegenüber einer Verfahrenslegitimität, die vorwiegend auf den Befriedigungswert der Entscheidungsbetroffenen zielt.

${ }^{18}$ WOLFGANG NAUCKE, Die Stilisierung von Sachverhaltsschilderungen durch materielles
} 
In diesem Zitat spiegelt sich letzthin der Sachverhalt wider, dass die Rechtsprechung die unsicheren, aber rechtsrelevanten Sachverhalte aus ihrer Umwelt (die , unformalisierte wirkliche Wahrheit') in ein systemeigenes Werte- und Normengeflecht einpassen muss, um sie nach Maßgabe eigener Entscheidungskriterien kommunizieren zu können. Die Inklusion des Falls in das Recht ist insofern gleichbedeutend mit dem Ausschluss systemfremder Entscheidungsreferenzen. Auf diesem Wege wird der Ursprungskonflikt in einen Rechtskonflikt überführt, mit der Folge, dass damit auch die Konfliktdynamiken des Ursprungskonflikts durch den Rechtsprozess abgelöst werden. ${ }^{19}$

Vor dem Hintergrund eigener Untersuchungen ${ }^{20}$ über die generischen Prozesse der Konfliktkonstitution möchte ich nachfolgend die These vertreten, dass die Sprache des Rechts und die Sprache des Konflikts komplementäre Beziehungen unterhalten. Diesen Überlegungen zufolge muss die Sprache des Rechts die Rhetoriken des Konflikts unterdrücken, um das Risiko destruktiver Konfliktentwicklungen vor Gericht vermeiden bzw. den Konflikt besser disputieren und entscheiden zu können. Die Sprache der rechtlich ,formalisierten wirklichen Wahrheit' ist demnach auch ein Reflex auf die unformalisierte Wahrheit sozialer Konflikte. Das wiederum heißt: Die typischen Formeigenschaften der rechtlichen Kommunikation haben - wenigstens teilweise - in den kommunikativen Merkmalen sozialer Konflikte ihre Wurzel. Dass zwischen Recht und Konflikt ein enger Sachzusammenhang existiert, muss man nicht eigens erörtern. Neu erscheint dagegen die Überlegung, dass die Sprache des Rechts auch als ein Umkehrbild sozialer Konfliktdynamiken aufgefasst werden kann und die kommunikativen Formen des Rechts die Muster der Konfliktkommunikation neutralisieren.

Zur näheren Bestimmung der Beziehung von Konflikt- und Rechtskommunikation möchte ich nachfolgend Aufschluss über die zentralen Strukturmuster der Konfliktgenese vermitteln. Dazu möchte ich zeigen, dass die Ausdifferenzierung sozialer Konflikte einheitlichen Entwicklungsdynamiken und Phasenabläufen folgt, die sich über die ihr jeweils

Strafrecht und Strafprozessrecht, in: JORG SCHONERT (Hrsg.), Erzählte Kriminalität (Fn. 2), $59,66$.

${ }^{19} \mathrm{Vgl}$. JOHANNES BRINKMANN, Konflikt, Konfliktregulierung und Recht: Ein vorläufiger Problemkatalog, in: Soziale Welt 24 (1973), 79; VOLKMAR GESSNER, Recht und Konflikt. Tübingen 1976; WILLIAM L. F. FELSTINER/RICHARD L. ABEL/AUSTIN SARAT, The Emergence and Transformation of Disputes: Naming, Blaming, Claiming, in: Law and Society Review 15 (1980), 631, für unterschiedliche Darstellungen dieses Vorgangs.

${ }^{20}$ Die nachfolgenden Überlegungen stützen sich auf meine Untersuchungen zu den generischen und reproduktiven Voraussetzungen sozialer Konflikte, vgl. HEINZ MESSMER, Der soziale Konflikt: Kommunikative Emergenz und systemische Reproduktion. Stuttgart 2003. 
zugrunde liegenden kommunikativen Formen begründen (2). Diese Formen werden sodann mit den Struktureigenschaften der Rechtskommunikation konfrontiert. In diesem Zusammenhang werde ich mich auf die Frage konzentrieren, wie die Kommunikation im Verfahren die Beteiligtenrollen im Konflikt neutralisiert und zudem die Formen konfliktpragmatischer Anschuldigungskommunikationen aufhebt (3). Die ,Feinmechanik' der Rechtskommunikation werde ich an einem kurzen Beispiel der gerichtlichen Zeugenvernehmung erläutern (4). Die kommunikationstheoretischen Analysen werden abschließend mit Annahmen zu den gesellschaftstheoretischen Funktionen des Rechts in Beziehung gesetzt (5)

\section{Konflikt und Sprache}

Konflikte werden - zuerst und vor allem - mit sprachlichen Mitteln ausdifferenziert. Sie sind nicht einfach nur, da', sondern Resultat einer spezifischen Operation des Sozialen. Der Prozess ihrer Ausdifferenzierung beginnt, ${ }^{21}$ wenn die Kommunikation einer Sinnzumutung auf Ablehnung stößt ("Leihst Du mir Deinen Wagen?" - "Nein."). Was immer das auslösende Sinnmoment (gegenläufige Erwartungen, Interessen, Bedürfnisse etc.) für die Ablehnung ist, es muss dem anderen mitgeteilt werden, damit dieser die Unvereinbarkeit mit dem je eigenen Standpunkt bemerken und darauf eingehen kann. Die empirische Realität sozialer Konflikte ist insofern Resultat einer kommunikativen Interaktion, deren charakteristisches Merkmal Widerspruch bzw. Ablehnung ist. In diesem Sinne gebrauchen wir nachfolgend den Terminus "Widerspruchskommunikation". Widerspruchskommunikationen sind zunächst einmal ubiquitäre und passagere Elementarereignisse der alltäglichen Interaktion. Sie zeigen die Möglichkeit sozialer Konfliktbildungen an, ohne sie deswegen auch schon zu exekutieren. Nicht jedes ,Nein' führt notwendigerweise auch zum Konflikt. Derjenige, der mit seinen Sinnzumutungen auf Ablehnung trifft, könnte seine Erwartung beispielsweise überdenken. Zur vollständigen Ausdifferenzierung und einer gewissen Stabilität des Konflikts kommt es vielmehr erst dann,

\footnotetext{
21 Die Grundannahmen der hier vorgestellten Theorie orientieren sich an den konflikttheoretischen Überlegungen von NIKLAS LUHMANN, Soziale Systeme: Grundriß einer allgemeinen Theorie. Frankfurt am Main 1984, 488 ff., weichen jedoch schon bald davon ab und führen darüber hinaus. Die gemeinsame These dieser Überlegungen aber ist, dass man soziale Konflikte analog zu den Ausdifferenzierungsprozessen sozialer Systeme nachzeichnen kann, wenn man unter Systemen einen Komplex von Operationen versteht, der sich kommunikativ reproduzieren und von nicht-dazugehörigen Umwelten abgrenzen kann.
} 
wenn sich die kommunizierten Sinnzumutungen wechselseitig negieren und beide Seiten sich darin unnachgiebig zeigen. Erst dann können diejenigen Erwartungsstrukturen ausdifferenziert werden, auf die der Konflikt seine (kommunikative) Reproduktion dauerhaft aufstützen kann.

Im Unterschied zu den sozialwissenschaftlich gängigen Konfliktparadigmen geht dieser Ansatz von der Annahme aus, dass Konfliktentwicklungen durch externe Einflussvariablen nicht angemessen erfasst und beschrieben werden können. Vielmehr sehen wir in der Ausdifferenzierung sozialer Konflikte einen binnengesteuerten Differenzierungsprozess, der sich über seine eigenen Operationen selbstbezüglich - gewissermaßen autistisch - konstituiert. Entsprechend ist auch die Konfliktstruktur das Produkt einer systeminternen Operation, die dadurch entsteht, dass beide Seiten beginnen, ihre Erwartungen an der Ablehnung des jeweils anderen $\mathrm{zu}$ orientieren. Nach Luhmann sind Widersprüche „im System selbst konstituierte Synthesen, Zusammenfassungen von Sinnmomenten unter dem Gesichtspunkt ihrer Unvereinbarkeit." 22 Wenn Alter unterstellt, dass Ego seine Sinnofferten ablehnen wird und Ego von ihm dasselbe erwartet, entsteht eine Negativversion doppelter Kontingenz, die beiden Seiten mithin verlässliche Verhaltensorientierungen bietet: "Ich tue nicht, was Du möchtest, wenn Du nicht tust, was ich möchte. "23 Die Negativversion doppelter Kontingenz ist vielleicht die formal grundlegenste Beschreibung einer Konfliktstruktur.

Ist die Unvereinbarkeit schließlich soweit gediehen, stellt sich die Frage, wie es im Anschluss daran weitergeht. Struktur gebend für die Reproduktionsfähigkeit sozialer Konflikte ist zunächst die Etablierung eines Themas, das der Widerspruchskommunikation eine inhaltliche Orientierung vermittelt. Konfliktthemen entstehen auf einem quasi ,natürlichen' Wege im Rahmen der Widerspruchskommunikation, wenn beide Seiten ihre jeweiligen Standpunkte mit Bezug auf den strittigen Sachverhalt zu klären versuchen. ${ }^{24}$ Im Zuge der Widerspruchskommunikation können die strittigen Themen wechseln, indem sich die Akzente unmerklich in andere Themenbereiche verlagern, was typischerweise dann geschieht, wenn man mit immer neuen Argumenten und Begründungen die Position des anderen zu erschüttern versucht

22 Vgl. LuHManN, Soziale Systeme (Fn. 21), 525.

23 Ebd., 531.

${ }^{24}$ Vgl. näher KaREN TRACY, Staying at Topic: An Explication of Conversational Relevance, in: Discourse Processes 7 (1984), 447; JOHN WILSON, On the Topic of Conversation as a Speech Event. Research on Language and Social Interaction 21 (1987), 93; KLAUS FOPPA, Topic Progression and Intention, in: IVANA MARKOVA/KLAUS FOPPA (Hrsg.), The Dynamics of Dialogue. New York 1990, 178. 
und infolgedessen immer neue Gesichtspunkte der Auseinandersetzung heranzitiert werden. Im Versuch, die je andere Seite trotz ihres Widerstands zum Nachgeben zu bewegen, sind die Voraussetzungen einer Themenausweitung strukturell immanent.

Sofern die Themen der Widerspruchskommunikation die soziale Beziehung der Konfliktbeteiligten unberührt lassen, bleibt das Konfliktgeschehen aus gleich näher ersichtlichen Gründen primär dem sachlichen Konflikterleben verhaftet. Sachlich ist eine Auseinandersetzung immer dann, wenn der Widerspruch auf einen Standpunkt und nicht auf die personale Identität der Konfliktbeteiligten zielt. Um zu signalisieren, dass sich die Ablehnung primär auf die Sache, nicht aber auf die Person des anderen richtet, werden im Rahmen der Widerspruchskommunikation häufiger unterschiedliche Formen sozialer Achtungserweise mitkommuniziert. ${ }^{25}$ Sachlich bedingte Unvereinbarkeit und soziale Harmonie schließen einander nicht notwendig aus. Sachkonflikte haben die Eigenschaft, dass sie den strittigen Sachverhalt mitunter mühelos verbreitern oder vertiefen, sofern jeder Beitrag zum Thema Anlass für weitere Ablehnungen gibt, jedoch können sie sich nur innerhalb eines abgesteckten Rahmens auch intensivieren - wenn man unter dem Prozess der Konfliktintensivierung die zunehmende Rigidität der Widerspruchsformen versteht. ${ }^{26}$ Dies ist zum Beispiel dann der Fall, wenn vormals mitkommunizierte Achtungserweise unterbleiben, die Tonlage kühler, oder die Ablehnung in rigideren Begriffen zum Ausdruck gebracht wird.

Die Prozessqualitäten des Sachkonflikts verändern sich in dem Augenblick grundlegend und radikal, wenn die Beziehung der Konfliktbeteiligten selbst zum vorherrschenden Gegenstand der Unvereinbarkeit wird, was soviel heißt, dass die Streitgegner selbst einander als Thema und Auslöseanlass für Ablehnung dienen. In diesem Fall ist nachfolgend von "Beziehungskonflikten“ die Rede. ${ }^{27}$ Allein mit den Mitteln der Widerspruchskommunikation ist dieses Stadium der Konfliktent-

25 Grundlegend dazu Penelope Brown/StePhen C. Levinson, Politeness: Some Universals in Language Usage. Cambridge 1987; ferner RICHARD J. WaTTS, Politeness. Cambridge 2003; für materiale Analysen vgl. MESSMER, Der soziale Konflikt (Fn. 20), 151 ff.

${ }^{26} \mathrm{Vgl}$. auch VIRGINIA W. COOPER, The Measurement of Conflict Interaction Intensity: Observer and Participant Perceived Dimensions, in: Human Relations 41 (1988), 171, 172.

27 Die Unterscheidung von Sach- und Beziehungskonflikten wurzelt in der pragmatischen Kommunikationstheorie von PAUL WATZlawICK/JANET H. BEAVIN/DON D. JACKSON, Menschliche Kommunikation: Formen, Störungen, Paradoxien. 6. Aufl., Bern 1982, 53 ff., der zufolge jede Kommunikation einen Inhalts- und einen Beziehungsaspekt hat, wobei Letzterer den Ersten bestimmt. Von der Form der Kommunikation hängt es ab, welcher Aspekt jeweils im Vordergrund der Mitteilung steht, ob also der Inhalts- oder der Beziehungsaspekt der Kommunikation zum Thema der Auseinandersetzung avanciert. 
wicklung jedoch nicht zu erreichen. Um den Sachkonflikt in einen Beziehungskonflikt $\mathrm{zu}$ transformieren, bedarf es vielmehr einer eigenständigen kommunikativen Form, die wir nachfolgend mit dem Begriff der "Anschuldigungskommunikation" näher bezeichnen.

Im Unterschied zur Widerspruchskommunikation verdichtet die Anschuldigungskommunikation verschiedene Teilkomponenten der $\mathrm{Zu}$ rechnung von Verantwortlichkeit zu einer kommunikativ eigenständigen und komplexen Formqualität. Dazu liegen mittlerweile (konversations-)analytisch schlüssige Forschungen vor, an denen sich auch die folgenden Überlegungen orientieren. ${ }^{28}$ Für die Anschuldigungskommunikation unverzichtbar ist erstens die Identifizierung eines Negativerlebens, das zur näheren Erforschung seiner Ursachen anregt (unhappy incident): Es zieht unangenehm, und man fragt sich, warum. Zweitens benötigt die Anschuldigung eine zum Verhalten befähigte Identität, die imstande ist, den als unerfreulich bemerkten Sachverhalt zu bewirken (actor-agent) - Hans beispielsweise. Drittens benötigt die Anschuldigung die Identifizierung einer vorausliegenden Handlung, die dem unhappy incident als Ursache dient (antecedent action): Eben war Hans noch im Zimmer und hat beim Hinausgehen die Tür nicht geschlossen. Und viertens muss der aus der Handlung des bezeichneten Akteurs hervorgegangene Sachverhalt mit dem unhappy incident in einer kausal offensichtlichen Beziehung stehen (consequent event). Während also der Widerspruchskommunikation das bloße ,Nein' als Bedingung ihrer operativen Wirksamkeit im Konflikt durchaus genügt, ist die Anschuldigungskommunikation demgegenüber auf das komplexe Zusammenspiel verschiedener Zurechnungskomponenten von Verantwortlichkeit angewiesen. Diese setzt den Wirkzusammenhang von Akteur und Ereignis voraus, dem sie darüber hinaus eine zeitliche Struktur unterstellt (antecedent action/consequent event). Das hört sich theoretisch komplizierter an als es in der Praxis umsetzbar ist. Man sagt beispielsweise: "Warum musst du immer die Tür offen lassen?" schon ist ein kommunikationsstrukturell anspruchsvolles Werk in die Tat umgesetzt.

Anschuldigungen rechnen soziale Verantwortung für unerfreuliche Ereignisse zu und zielen auf Schuldanerkennung, Schadenswieder-

\footnotetext{
${ }^{28}$ So z. B. PAUL DREW, Accusations: The Occasioned Use of Members' Knowledge of ,Religious Geography' in Describing Events, in: Sociology 12 (1978), 1; KELLY G. SHAVER, The Attribution of Blame: Causality, Responsibility, and Blameworthiness. New York 1985; RICHARD BUTTNY, Social Accountability in Communication. London 1993; IAN DERSLEY/ANTHONY WOOTTON, Complaint Sequences Within Antagonistic Argument, in: Research on Language and Social Interaction 33 (2000), 375; die obige Darstellung orientiert sich vorwiegend an den prägnanten Überlegungen von ANITA POMERANTZ, Attributions of Responsibility: Blamings, in: Sociology 12 (1978), 115.
} 
gutmachung und gegebenenfalls auf die Legitimität einer Sanktion. Sie wollen mehr als nur überzeugen. Sie wollen ein konkretes Verhalten verändern, sofern dieses unangenehme bzw. sozial inakzeptable Folgen nach sich zieht. Dieser Anspruchslage entsprechend wird mit der Anschuldigung eine Konfliktform inauguriert, die meist so schwer wiegt, dass sie sich der Aufmerksamkeit des anderen sicher sein kann, mithin schon deswegen, als sie dessen positives Selbstbild bedroht. ${ }^{29}$ Wer sich daher im Konflikt auf die Rhetoriken der Anschuldigung stützt, wird mit hoher Wahrscheinlichkeit auf beträchtlichen Widerstand treffen. Als Komplement einer feststehenden Paarbeziehung ${ }^{30}$ wird vor allem die Rechtfertigung kommunikationsstrukturell virulent. ${ }^{31}$ Defensive Rechtfertigungsstrategien zielen auf die Entkräftung des Unrechtscharakters des inkriminierten Verhaltens, während offensive Rechtfertigungsstrategien ihrerseits Schuld und Verantwortung auf die Gegenseite attribuieren, also mit Gegenanschuldigung kontern. Symmetrie in der Anschuldigungskommunikation (Anschuldigung/Gegenanschuldigung) hat auf Dauer zur Folge, dass sie den Konflikt als Beziehungskonflikt reproduziert.

Ursache und Anlass für Gegenanschuldigungen wurzeln weniger in den einen Konflikt auslösenden Streitsachverhalten, sondern vor allem in den sich daran anschließenden Prozessen der Anschuldigungskommunikation. Sie sind maßgebliche Aspekte der Konflikteskalation und ihren Prozessen endemisch. In dem Maße zum Beispiel, wie man den Konflikt als psychische oder soziale Belastung erfährt, ist man geneigt, seinen Unmut auf die gegnerische Identität zu konzentrieren, der man damit die Schuld an der vorliegenden Misere attestiert. Sofern dieser Vorgang sich als vorherrschendes Muster der Konfliktinteraktion etabliert, werden sich beide Seiten zunehmend in regenerative Schuldzuschreibungszirkel verstricken. Auch dieses Muster besitzt eine den

$29 \mathrm{Vgl}$. BARRY R. SCHLENKER, Impression Management: The Self-Concept, Social Identity, and Interpersonal Relations. Monterey 1980; ferner THOMAS J. SCHEFF, Shame and Conformity: The Deference-Emotion System, in: American Sociological Review 53 (1988), 395; WAYNE A. BEACH, Avoiding Ownership For Alleged Wrongdoings. Research on Language and Social Interaction 24 (1990/91), 1. Gemeinsam ist diesen Analysen die Einsicht, dass man ohne positive Selbstsicht auf Dauer nur schwer zurechtkommt; klassisch dazu: LeON Festinger, A Theory of Cognitive Dissonance. Stanford 1957; Ders., Conflict, Decision, Dissonance. Stanford 1964.

30 Die Konversationsanalyse benutzt hierfür den Begriff des adjacency pair: Gemeint sind kommunikativ reziproke Gattungsbeziehungen wie Gruß/Gegengruß, Einladung/ Annahme (Ablehnung), Vorwurf/ Entschuldigung, etc.

$31 \mathrm{Vgl}$. dazu die von BUTTNY, Social Accountability in Communication (Fn. 28), 38, herausdestillilierte self defense rule: "This rule interactionally provides for a slot after the blame for the accused to respond to critics (...): upon receiving a blame, make a response (...) lest no response be heard by others as an admission to the blame." 
Prozess in der Sozialdimension des Konflikts stabilisierende Struktur, die wir im Anschluss an die pragmatische Kommunikationstheorie mit dem Terminus der "Interpunktion" 32 bezeichnen.

Interpunktion ist ein für den Beziehungskonflikt zentraler Strukturparameter, durch den sich die Erwartungen der Beteiligten in der Art einer self-fulfilling prophecy ineinander verketten. Durch Interpunktion wird das individuelle Konflikterleben so strukturiert, dass jeweils der andere ursächlich als Anlass immer weiterer Konfliktepisoden aufscheint, auf die man selbst - scheinbar gezwungenermaßen - mit Gegenmaßnahmen reagiert. So erscheint Egos Handeln einem Alter als Ursache für seine eigene Reaktion, diese wiederum ist Anlass für eine Reaktion Egos, die Alter seinerseits wieder als ursächliches Handeln interpretiert. Auf kommunikationsstruktureller Ebene ist die Anschuldigung des einen Anlass (unhappy incident) für die Gegenanschuldigung des anderen, diese wiederum für Gegenanschuldigung des einen, und so weiter als infiniter Regress. Aus analytischer Sicht werden die Reiz/Reaktionsbeziehungen gemäß Anschuldigung und Gegenanschuldigung jeweils so miteinander verknüpft, dass jeweils die andere Seite kausal ursächlich für die eigenen Reaktionen verantwortlich gemacht werden kann.

Konflikttheoretisch hat die Reproduktion der Unvereinbarkeit im Medium der Anschuldigungskommunikation für die Konfliktbeteiligten weit reichende Folgen. Indem die Anschuldigung des einen zum unhappy incident des anderen wird, verlagert der Konflikt seinen Schwerpunkt von der Sach- in die Sozialdimension sinnhaften Erlebens. ${ }^{33}$ Die Verständigungs- und Empathiebereitschaft beider Seiten nimmt auf Grund fortlaufender Anschuldigungen signifikant $a b$, während selbstreferenzielle und autologische Sinnorientierungen an Bedeutung gewinnen. Die Wahrnehmungen im Hinblick auf das Verhalten

\footnotetext{
32 Das Konzept der Interpunktion beruht auf der Überlegung, dass jede Mitteilung Reiz und Reaktion für ein bestimmtes Verhalten ist. Sofern die eine Seite ein Verhalten als Reiz, die andere dieses als Reaktion auffasst, werden ineinander fließende Interaktionsverkettungen ungleich interpunktiert. Entwickelt wurde diese Idee von GREGORY BATESON/DON D. JACKSON, Some Varieties of Pathogenic Organization, in: DAVID MCK. RIOCH (Hrsg.), Disorders of Communication. Association for Research in Nervous and Mental Disease. Research Publications Vol. 42 (1964), 270, 273. Sie nimmt als drittes Axiom bei WATZLAWICK/BEAVIN/JACKSON, Menschliche Kommunikation (Fn. 27), 57 ff., einen wichtigen Platz in der pragmatischen Kommunikationstheorie ein, wo sie pathologische Entwicklungsverläufe in zwischenmenschlichen Beziehungsdynamiken erklären soll.

33 "In der Sozialdimension", so LUHMANN, Soziale Systeme (Fn. 21), 125, „werden Ego und Alter für Zurechnungszwecke personalisiert bzw. mit bestimmten sozialen Systemen identifiziert. Sie erhalten, ungeachtet ihres jeweiligen Fungierens als Ego und als Alter für ein alter Ego, Identitäten, Namen und Adressen. “
} 
des anderen werden auf diesem Wege zunehmend inadäquat und mithin zu einem Feindbild verdichtet. Anders als in der (sachlichen) Widerspruchskommunikation stellt die Anschuldigung nicht nur die einzelne Sinnzumutung zur Disposition, sondern die personale und moralische Integrität des jeweils anderen. Einzelhandlungen werden auf diesem Wege zunehmend indexikalisch. ${ }^{34}$

Neben dem Wechsel von der Widerspruchs- zur Anschuldigungskommunikation überschreitet die Ausdifferenzierung sozialer Konflikte unter Umständen noch eine dritte bedeutsame Schwelle. Wenn die Durchsetzung von Standpunkten weder auf dem Wege des Überzeugens noch mit den Mitteln der Schuldzuschreibung gelingt, stehen den Beteiligten noch Macht und Gewalt als Durchsetzungsmittel zur Verfügung. Macht und Gewalt sind ultimative Konfliktstrategien, um den Widerstandswillen des anderen $\mathrm{zu}$ brechen. Damit eine Unvereinbarkeit auf diese Ebene transferiert werden kann, müssen die Kommunikationsstrukturen im Konflikt jedoch erneut umgepolt werden. Typisch für Machtkonflikte ist die Drohkommunikation, die sich in Form einer negativ bewerteten Vermeidungsalternative ganz auf den gegnerischen Widerstandswillen konzentriert: Wenn du nicht , $X^{\prime}$ - so sagt der Drohsteller - dann erfolgt , $Y^{\prime}$, wobei , $X^{\prime}$ das erwünschte, aber bislang verweigerte Verhalten und , $Y^{\prime}$ den Einsatz in Aussicht gestellter, aber bislang noch unausgeführter Machtmittel repräsentiert. ${ }^{35}$

Drohungen sind konditional strukturierte Muster der Konfliktkommunikation, die mit Überlegenheitsansprüchen operieren. Angesichts fruchtlos gebliebener Durchsetzungsbemühungen sind Drohungen insofern attraktiv, weil sie dem Drohsteller für verhältnismäßig wenig Aufwand einen hohen Nutzen versprechen. ${ }^{36}$ Sie sind - vorausgesetzt

\footnotetext{
34 Dazu schreibt ERVING GOFFMAN, Das Individuum im öffentlichen Austausch: Mikrostudien zur öffentlichen Ordnung. Frankfurt am Main 1982, 140 f.: "Durch die Tendenz der Individuen, einzelne Handlungen als etwas Symptomatisches aufzufassen, erhalten sogar Handlungen ganz eigenständiger Art eine beträchtliche indikatorische Bedeutung. Sie werfen ein Licht auf die allgemeine Beziehung des Akteurs zu einer Regel und darüber hinaus auf seine Beziehung zu dem Regelsystem, von dem die fragliche Regel bloB ein Teil ist. Natürlich wird eine solche Information oft auch als relevant für die Beurteilung des moralischen Charakters des Akteurs angesehen"; vgl. auch D. RODNEY WATSON, Categorization, Authorization, and Blame-Negotiation in Conversation, in: Sociology 12 (1978), 105 ff; mit Bezug auf Moral: JÖRG R. BERGMANN/THOMAS LUCKMANN, Moral und Kommunikation, in: DIES. (Hrsg.), Kommunikative Konstruktion von Moral. Band 1: Struktur und Dynamik der Formen moralischer Kommunikation. Opladen 1999, 15 ff.

$35 \mathrm{Vgl}$. beispielsweise RAINER PARIS/WOLFGANG SOFSKY, Drohungen: Über eine Methode der Interaktionsmacht. Kölner Zeitschrift fïr Soziologie und Sozialpsychologie 39 (1987), 15.

36 "Something for nothing" heißt es beispielsweise bei CAROL M. RUBIN/JEFFREY Z. RUBIN, Dynamics of Conflict Escalation in Families: The Role of Threats, Promises, Guilt Induction, and Passive Manipulation, in: Daniel Perlman/Warren H. JONes (Hrsg.)
} 
sie gelingen - ein negatives und deshalb ein umso vorteilhafteres Tauschgeschäft. ${ }^{37}$ Auf diesem Wege werden die noch bestehenden Elemente kommunikativer Verständigungsmöglichkeiten allerdings radikal eliminiert. Wer droht, der kündigt (und sei es nur für einen kurzen Moment) sein Restkontingent an Rücksichtnahme für den anderen auf und setzt sich über Argumente, Erklärungen und Rechtfertigungen rigide hinweg. Entsprechend wird der Konflikt auf ein Nullsummenkalkül reduziert, das außer Durchsetzen und Nachgeben keine anderen Alternativen mehr kennt. Der Drohbetroffene kann allenfalls noch versuchen, mittels Gegendrohung zu überzeugen, in der Hoffnung, den Drohsteller seinerseits einzuschüchtern und ihn an der Ausführung seines Drohversprechens zu hindern. Drohungen werden mit Blick auf Durchsetzung konzipiert, sie besitzen eine hohe Selbstbindungskraft und sind entsprechend schlecht $\mathrm{zu}$ verhandeln. In diesem Sinne markiert die Drohkommunikation das obere Ende der kommunikativ inszenierten Konflikteskalation. Im Drohfall ist Kommunikation dispräferiert, das heißt, gute Gründe' sind für die Drohabwehr weder angemessene noch Erfolg versprechende Mittel. Unter diesen Voraussetzungen werden mitunter umfassende Drohsysteme ausdifferenziert, deren Logik auf einer progressiven Steigerung der Abschreckungsintensität fußt. ${ }^{38}$

Auf der Grundlage der hier nur verkürzt dargestellten Analysen lassen sich die operativen Formen der Ausdifferenzierung sozialer Konflikte auf wenige Grundprinzipien des Ablehnens reduzieren. Jedes Einzelne drückt dem Konfliktgeschehen unverwechselbar seinen Stempel auf. Mit der Widerspruchskommunikation werden Unvereinbarkeiten als themengesteuerte Sachkonflikte prozessiert, die sich zwar unbegrenzt ausweiten können, den Konfliktgegner in seiner Form als Person thematisch aber unberücksichtigt lassen. Dieser erhält erst durch die Anschuldigungskommunikation seine zentrale Bedeutung für den Konflikt. In diesem Fall steht das soziale Beziehungserleben zur Disposition, sofern die Schuldzurechnung vorwiegend den sozialen Antagonismus vertieft. Die Drohkommunikation wiederum setzt sich über die Anerkennung von Interessen und Bedürfnissen rigide hinweg und ignoriert auch die Form der Person. Sie konzentriert sich stattdessen ganz auf den Widerstandswillen des anderen, soweit dieser den eigenen Durchsetzungsabsichten im Wege steht. Die durch die Drohkom-

Advances in Personal Relationships. Vol. 4. London 1993, 165, 172, die damit die Attraktivität und den Erfolg einer Drohung als kostengünstigen Tauschfaktor bestimmen.

37 So auch BERNHARD KÜLP, Theorie der Drohung. Köln 1965, 42 f.

38 Vgl beispielsweise KENNETH E. BOULDING, Towards a Pure Theory of Threat Systems, in: American Economic Review 53 (1963), 424. 
munikation erzeugte Konfliktrealität rechtfertigt es, in diesem Fall von Machtkonflikten zu sprechen.

Widerspruchs-, Anschuldigungs- und Drohkommunikationen reproduzieren den Konflikt auf je unterschiedlichen Niveaus seiner Ausbreitung und Intensität. Sie bezeichnen die elementaren Schwellenprozesse in der Ausdifferenzierung des Konflikts, die seine Entwicklung vergleichbar mit dem Differential eines Motorengetriebes - mit jedem Schaltvorgang auf einer höheren Ebene seiner Emergenz beschleunigt reproduzieren. Empirisch lässt sich der Prozess der Konflikteskalation mit den hier beschriebenen kommunikativen Typen mühelos rekonstruieren - ausgehend vom konfliktinduzierenden 'Nein' bis hin zum Abbruch der Konfliktkommunikation und der Fortführung des Konflikts mit physischen Mitteln. Eine einfache Vorstellung der hier skizzierten Konflikttheorie vermittelt die nachfolgende Abbildung 1, die den Konfliktprozess in Form eines Treppenmodells illustriert:

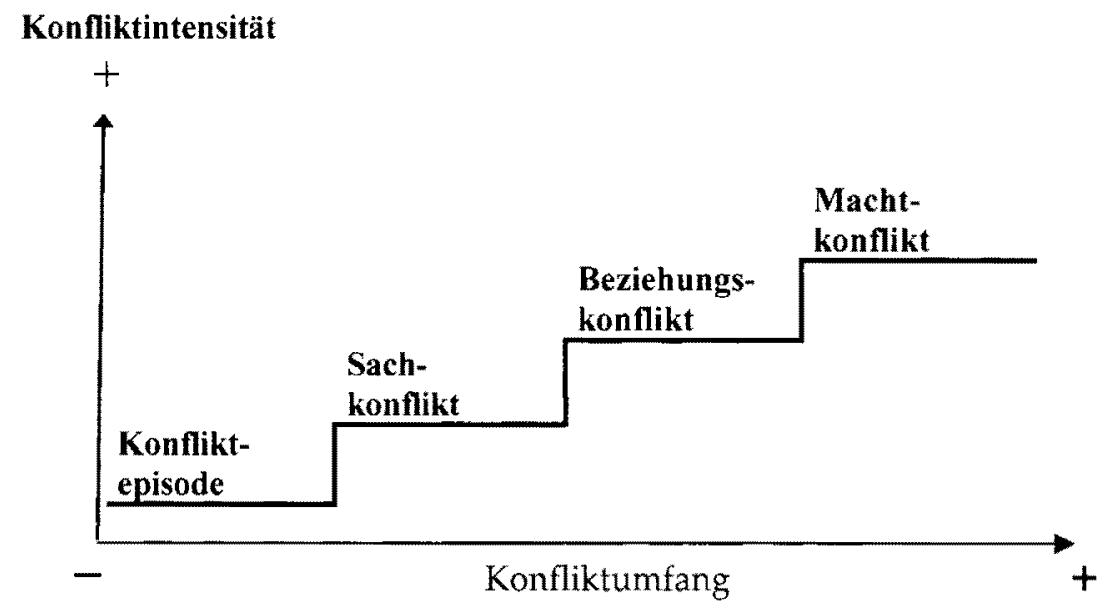

Abb. 1: Prozessstufenmodell der Ausdifferenzierung sozialer Konflikte

Aus kommunikationspragmatischer Sicht sind die einzelnen Etappen der Ausdifferenzierung sozialer Konflikte also Resultat verschiedenartiger Verweismöglichkeiten der Konfliktkommunikation. Jede Abwandlung im Gebrauch kommunikativ verfügbarer Durchsetzungsstrategien verändert die Beziehungsmuster im Konflikt in bedeutsamer Weise. Prozesse zunehmender Konfliktintensität sind im Allgemeinen gleich- 
bedeutend mit dem Rückgang sozialer Empathiefähigkeit. Sie erodieren die Grundlagen sozialer Kooperation und führen die Konfliktgegner zunehmend auseinander. Diese werden mit fortschreitender Konfliktentwicklung jedoch auch immer tiefer in die Konfliktbeziehung verstrickt. Die Ausdifferenzierung des Konflikts bindet nicht nur immer mehr Themen an die Widerspruchskommunikation, sondern greift mithin auf die persönliche Selbstachtung und physischen Machtungleichgewichte der Beteiligten über, die man schließlich nicht mehr ohne weiteres ignorieren oder einfach nur stehen lassen kann.

\section{Rechtliches Konfliktmanagement}

Auslöseanlass für das In-Kraft-Treten des Rechts ist der Dissens zweier Seiten bzw. ein Konflikt zwischen Individuum und rechtlicher Norm. Dem Dissens kann eine privatrechtliche Streitursache zugrunde liegen oder auch eine Vertragsverletzung, bei der die Rechtsgeltung strittig ist. Gleiches gilt für den strafrechtlichen Sachverhalt, unabhängig von der Frage, ob von einem inkriminierten Verhalten Personen als Opfer betroffen sind. In diesem Fall übernimmt die Staatsgewalt die Position des Opfers und Klägers, die auf die Diskrepanz von Normvorschrift und inkriminierten Verhalten aufmerksam macht. ${ }^{39}$ Mithin wird das

\footnotetext{
${ }^{39}$ Aus konflikttheoretischer Sicht drängt sich hier im Übrigen die Vermutung auf, dass Gewaltverbrechen aus Beziehungskonflikten resultieren und in Form von Machkonflikten eskalieren, bis sie schließlich gewaltsam beendet werden; für Hinweise in diese Richtung vgl. ALBERTO GODENZI, Gewalt im sozialen Nahraum. Basel 1993; ROY BAUMEISTER/ARLENE M. STILlWEll/TOdD F. HEATHERTON, Interpersonal Aspects of Guilt: Evidence From Narrative Studies, in: JUNE PRICE TANGNEY/KURT W. FISCHER (Hrsg.), Self-Conscious Emotions: The Psychology of Shame, Guilt, Embarrassment, and Pride. New York 1995, 255; HeLMUT TAUSENDTEUFEL, Die Kehrseite romantischer Liebe: Intimpartnertötungen in West-Berlin in den Jahren 1950-1989. Diss. FU Berlin 1997; ROY F. BAUMEISTER/BRAD J. BUSHMAN, Emotion und Aggressivität, in: WILHELM HEITMEYER/JOHN HAGAN (Hrsg.), Internationales Handbuch der Gewaltforschung. Wiesbaden 2002, 598. Verbrechen erscheinen in dieser Perspektive gewissermaßen als destruktive und sozial missbilligte Formen des Konfliktaustrags. Aber auch Verbrechen ohne vorhergehende Beziehungsgeschichte lassen sich nachträglich als Konflikt rekonstruieren, etwa im Sinne einer interpunktierten Konfliktwirklichkeit, in der sich der Verbrecher weniger als Täter denn als Opfer erlebt, vgl. JEROME KATZ, The Seductions of Crime: Moral and Sensual Attractions in Doing Evil. New York 1988; ferner SUZANNE UNIACKE, Permissible Killing: The Self-Defence Justification of Homicide. Cambridge 1994. Diesen Vorstellungen liegt mithin die Möglichkeit zugrunde, dass man Normvorschriften kognitiv neutralisieren und sich selbst als Ausnahme von der Regel auffassen kann. Der andere, wer auch immer zum Opfer der eigenen Handlungen wird, hat es demnach nicht besser verdient; vgl. grundlegend: GRESHAM M. SYKES/DAVID MATZA, Techniques of Neutralization: A Theory of Delinquency, in: American Sociological Review 22 (1957), 664; DAVID MATZA, Becoming Deviant. Englewood Cliffs 1969. Für eine
} 
Recht durch unbeteiligte Dritte angerufen. ${ }^{40}$ Der take off rechtlicher Aktivitäten begründet sich ungeachtet der Verschiedenartigkeit ihrer Auslöseanlässe in allen Fällen gleich über die Zurechnungskomponenten der Anschuldigungskommunikation, auf die sich auch die Auseinandersetzung über Rechtsfragen im Weiteren stützt.

Der Konflikt wird also als ein Beziehungskonflikt in das Recht eingeführt und dort unter rechtseigenen Entscheidungsgesichtspunkten weiterbehandelt. ${ }^{41}$ Für die Parallelisierung von Rechts- und Beziehungskonflikt sprechen verschiedene logische Gründe. Analog zur binären Codierung des Rechts (Recht/Unrecht) weist der Beziehungskonflikt binär schematisierte Beteiligtenrollen als zentrales Konfliktthema aus (Ich/Du), die sich der einen oder anderen Seite des Rechtscodes entsprechend zuordnen lassen. Welche Seite im Recht und welche im Unrecht ist, wird damit zu einer Frage der empirischen Konkretion, die in den Verfahren des Rechts erst noch festgestellt und geklärt werden muss. Mit der rechtlichen Zuschreibung von Beteiligtenrollen (Kläger/Beklagte) ist der Rechtskonflikt allein noch nicht entschieden. Kläger können sich irren oder täuschen und Beklagte hinsichtlich des Schuldvorwurfs unschuldig sein. Das Gericht hält sich die Entscheidung nach Maßgabe eigener Urteilskriterien bis zum Richterspruch offen und vermeidet sorgfältig alle Hinweise auf Parteilichkeit. Nahe liegend ist die Vorstellung einer Kongruenz von Rechts- und Beziehungskonflikt zudem auf Grund ihrer kommunikativ identischen Formen.

empirische Analyse dieses Zusammenhangs: HEINZ MESSMER, Unrechtsaufarbeitung im Täter-Opfer-Ausgleich: Sozialwissenschaftliche Analysen zur außergerichtlichen Verfahrenspraxis bei Jugendlichen. Bonn 1996, $103 \mathrm{ff}$.

40 Auch TORSTEIN ECKHOFF, Die Rolle des Vermittelnden, des Richtenden und des Anordnenden bei der Lösung von Konflikten, in: ERNST HIRSCH/MANFRED REHBINDER (Hrsg.), Studien und Materialien zur Rechtssoziologie. Opladen 1971, 243, 247, unterscheidet vergleichbare Konfliktkonstellationen beim Inkrafttreten des Rechts: „Erstens sind Fälle denkbar, in denen keine der Parteien ein Interesse an der Lösung des Konflikts hat, wo aber ein Dritter oder die Umwelt sie von ihnen fordert (...) Zweitens kommt es oft vor, dass eine Partei, nicht aber die andere, an einer Lösung des Konflikts interessiert ist (...) Eine dritte Situation (...) entsteht, wenn beide Parteien an einer Lösung des Konflikts interessiert sind."

41 Damit wenden wir uns gegen eine weit verbreitete Ansicht, die zwischen Konflikten der Gegensätzlichkeit (von Interessen) und Konflikten der Nichtübereinstimmung (von Werten) unterscheidet, vgl. VILHELM AUBERT, Competition and Dissensus: Two Types of Conflict Resolution, in: The Journal of Conflict Resolution 7 (1963), 26, sowie DERS., In Search of Law: Sociological Approaches to Law. Totowa 1983, 63 ff., wo Aubert die Ansicht vertritt, dass vorwiegend Wertekonflikte förmliche Konfliktentscheidungsverfahren nach sich zögen, wohingegen Interessenskonflikte besser auf dem Wege der Aushandlung beigelegt würden. Diese Vorstellung ist analytisch und empirisch nicht überzeugend; kritisch dazu auch ECKHOFF, Die Rolle des Vermittelnden (Fn. 40), 244 ferner Philip H. Gulliver, Disputes and Negotiations: A Cross-Cultural Perspective. New York 1979,9 
Hier wie dort stützen sich die Verfahrensbeteiligten auf die elementaren Komponenten der Anschuldigungskommunikation, die sie mittels offensiver oder defensiver Rechtfertigungsstrategien untermauern.

Es besteht insofern eine gewisse Isomorphie von Beziehungs- und Rechtskonflikt im Hinblick auf die Separation der Parteien sowie auf die sprachlichen Äußerungen in Form einer Anschuldigungskommunikation. Der maßgebliche Unterschied zwischen beiden Varianten des Konfliktmanagements liegt indes in der Verschiedenartigkeit kommunikativer Referenzen: Nicht die eigene Überzeugung entscheidet über ,richtig' oder ,falsch' im Konflikt, sondern die Sichtweise des Gerichts, das sich allein der rechtlichen Norm bzw. dem Entscheidungsprogramm rechtlicher Verfahren verpflichtet und sich dem Kalkül der Entscheidungsbetroffenen dadurch entzieht. Aus denselben Gründen liegt auch die Wahl der Konfliktthemen nicht mehr länger im eigenen Ermessen, vielmehr erhält die Konfliktkommunikation auf Grund zahlreicher Thematisierungsschwellen vor Gericht ein ganz anders gelagertes Mitteilungsprofil: Andere bestimmen, wer wann in welchem Umfang zu welchen Themen Stellung beziehen kann. Indem das Gericht die Beteiligtenrollen, ihre Stellung im Konflikt sowie die Themen der Verantwortungszurechnung verfahrensstrukturell umarrangiert, schafft es damit die Voraussetzungen für ein kommunikatives Setting, das die binären Strukturen der Anschuldigungskommunikation grundsätzlich beibehält, ihre Rahmenbedingungen jedoch entscheidend verändert. Diesen Zusammenhang verdeutlicht nachfolgend Abbildung 2:
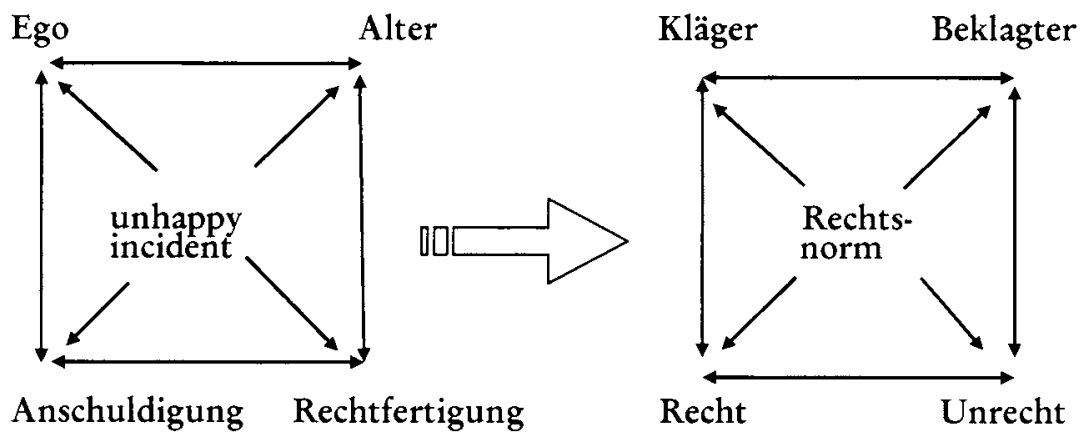

Abb. 2: Transformation sozialer Konflikte durch die rechtliche Norm

Im Mittelpunkt der Transformation vom Ursprungs- in den Rechtskonflikt steht demzufolge die Unterwerfung des subjektiven Konflikterlebens unter die rechtliche Norm. Kummer und Frustration, persönliches 
Ungerechtigkeitserleben oder gefühite Feindseligkeiten als lebensweltlich zentrale Komponenten der offenen Konflikteskalation ${ }^{42}$ werden vor Gericht als eher irrelevante Entscheidungsfaktoren behandelt. Verbrechensopfer zum Beispiel finden vor Gericht vornehmlich in ihrer Zeugeneigenschaft, weniger dagegen in ihrer Opferrolle Gehör. ${ }^{43}$ Aus rechtlicher Sicht ist das Opfererleben nicht schon per se indikatorisch für einen Verstoß gegen die Norm (wie immer die Betroffenen selbst darüber denken), sondern allenfalls Anlass für einen Prüfvorgang, der sich auf rechtsrelevante Sachverhaltsfeststellungen stützt.

Unter den Auspizien der rechtlichen Norm werden zunächst also die Beteiligtenrollen, und damit verbunden: die interaktiven Beziehungsdynamiken im Konflikt unterlaufen. Dieser Vorgang korrespondiert mit der blockierten Direktkommunikation zwischen Kläger und Beklagtem. Beispielsweise sind weder Klage noch Klageerwiderung direkt an die gegnerische Streitpartei adressiert, sondern werden zuerst dem Gericht zur Kenntnis gebracht. Bereits damit wird die unmittelbare Reaktivität von Anschuldigung und Gegenanschuldigung unterlaufen und in strukturell kontrollierte Bahnen gelenkt. Auch ist in der Gerichtskommunikation kein persönlicher Direktaustausch zwischen den Streitbeteiligten vorgesehen. Das ist prozessrechtlich zwar nicht von vornherein ausgeschlossen, in der Praxis aber eindeutig dispräferiert: Die Durchsetzung von Standpunkten wird typischerweise den prozessbevollmächtigten Anwälten überlassen. Der Interessensgegensatz bleibt dadurch erhalten bzw. wird durch die Prozessvertreter mithin noch beträchtlich geschürt, die Beziehungsdynamik der Ursprungskonflikts damit jedoch ausgebremst und in die Form professionell distanzierter Beteiligtenrollen überführt. Der gerichtliche Kommunikationsprozess läuft insofern über die Konfliktbeteiligten hinweg und an ihnen vorbei, die Kommunikationskanäle des Ursprungskonflikts versanden. Die Konfliktbeziehung wird damit im eigentlichen Wort-

$42 \mathrm{Vgl}$ als repräsentatives Beispiel jüngerer Überlegungen zu diesem Thema: OMAR J. BARTOS/PAUL WEHR, Using Conflict Theory. Cambridge 2002, 81, die eine Gemengelage von objektiven und subjektiven Einflüssen identifizieren, die hinreichend für den Beginn eines offenen Konfliktaustrags sind. Daraus lässt sich schließen, dass besonders die subjektiven, für das Feindseligkeitserleben der Konfliktbeteiligten unmittelbar mabgeblichen Gefülsfaktoren (Grievances/Frustration) vor Gericht an Einfluss verlieren.

43 Vgl. für daran anschließende Probleme: THOMAS WEIGEND, Deliktsopfer und Strafuerfahren. Berlin 1989; JO-ANNE WEMMERS, Victims in the Criminal Iustice System. Amsterdam 1996; ERHART KORTING, Justiz und Opferschut, in: Recht and Politik 35 (1999), 230 HOLCER HAUPT/ULRICH WEBER (Hrsg.), Handbuch Opferschutz und Opferhilfe. 2. Aufl Baden-Baden 2003; zu möglichen Alternativen im Rahmen von Mediation: MARTN WRIGHT, The Court as Last Resort: Victim-Sensifive, Community-Based Responses to Crime, in: The British Joumal of Criminology 42 (2002), $654 \mathrm{ff}$. 
sinn kaltgestellt, die Entscheidungsbetroffenen zum Publikum in eigener Sache, während die Parteienvertreter den Konflikt unter gerichtseigenen Prämissen neu konstituieren:

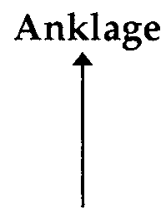

Ego
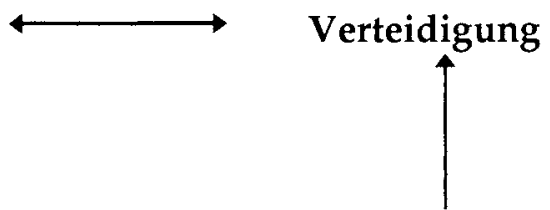

Alter

Abb. 3: Transformation sozialer Konflikte durch Parteienvertretung

Ein weiterer, konflikttheoretisch weit wichtigerer Aspekt der Neutralisierung interaktiver Konfliktdynamiken kommt aber erst mit der Einführung eines übergeordneten Dritten in das Entscheidungsverfahren zustande. ${ }^{44}$ Während die anwaltlichen Parteienvertreter den Interessenswiderspruch auf die rechtliche Norm fokussieren, wird mit Einführung eines Dritten in den Entscheidungsvorgang die bilaterale Konfliktbeziehung radikal destruiert. Denn von dessen ,Inbegriff der Überzeugung' hängt ab, welche Sachverhaltsdarstellung in welchen Umfang anerkannt wird. Den konfliktbedingten Erwartungssicherheiten einer bilateralen Gegnerschaft wird damit der Boden entzogen, sofern offen ist, welcher Darstellung der Richter folgen wird. Er ist idealiter weder als Freund noch als Feind zu betrachten, vielmehr muss man ihn erst zum einen oder anderen machen. Es reicht daher nicht mehr aus, allein

\footnotetext{
${ }^{44}$ Zur Theorie des Dritten als Konfliktlösungsparadigma besteht eine reichhaltige Literatur, vgl. etwa JOHAN GALTUNG, Institutionalized Conflict Resolution: A Theoretical Paradigm, in: Journal of Peace Research 4 (1965), 348; ORAN R. YOUNG, The Intermediares: Third Parties in International Crisis. Princeton 1967; RICHARD E. WALTON, Interpersonal Peacemaking: Confrontations and Third Party Consultation. Reading (Mass.) 1969; RONALD J. FISHER, Third Party Consultation: A Method for the Study and Resolution of Conflict, in: Journal of Conflict Resolution 16 (1972), 67, um einige frühe, aber einschlägige Beispiele zu nennen. Viele Überlegungen zum konfliktneutralen Dritten gehen auf die bahnbrechenden Analysen von GEORG SIMMEL, Soziologie: Untersuchungen ü̈ber die Formen der Vergesellschaftung. 6. Aufl., Berlin 1983, 75 ff. zurück, der wesentliche Bestimmungsunterschiede sozialer Gruppen aus der Zwei- oder Dreiwertigkeit ihrer Beziehungsmuster schöpft. Der Punkt, auf den es hier jedoch zuerst und vor allem ankommt: Jede Triade besitzt eine Tenden z zur Koalition von zwei gegen einen. $\mathrm{Zu}$ den Formen des Koalierens innerhalb der Triade vgl. THEODORE CAPLOW, A Theory of Coalitions in the Triad, in: American Sociological Review 21 (1956), $489 \mathrm{ff}$.
} 
den gegnerischen Standpunkt zu entkräften, sondern die Entkräftung muss darüber hinaus überzeugen. Das wiederum hat zur Folge, dass die Kommunikation vor Gericht im Unterschied zu der des Ursprungskonflikts viel stärker auf Selbstinszenierung ausgelegt ist, die einen unbeteiligten Dritten beeindrucken soll.

Kommunikationsstrukturell beruht das gerichtliche Konfliktmanagement auch diesbezüglich auf ganz anderen Entscheidungsprämissen als der Ursprungskonflikt. Zum einen wird die Konfliktkomplexität durch Einführung des Dritten beträchtlich erhöht, zum anderen die Kontrolle über das Konfliktmanagement externalisiert und in fremde Hände gelegt: Die im Grundsatz bilaterale Kommunikationsstruktur des Konflikts wird vor Gericht in ein trianguliertes Kommunikationsarrangement umgeformt, das vorwiegend Prozessbevollmächtigte und Richter bestreiten, während die Entscheidungsbetroffenen selbst nur noch unter speziellen Bedingungen darin berücksichtigt werden. Aus konflikttheoretischer Sicht ist bemerkenswert, dass sich mit Einführung der Dritten Partei ein Kommunikationssetting ausdifferenziert, das unter weitgehendem Ausschluss der Konfliktbetroffenen die kommunikative Ebenenvielfalt des Verfahrens beträchtlich erhöht, indem es den Richtungssinn bestehender Kommunikationsmöglichkeiten praktisch verdreifacht. Diesen Zusammenhang verdeutlicht Abbildung 4:

Ego

Alter

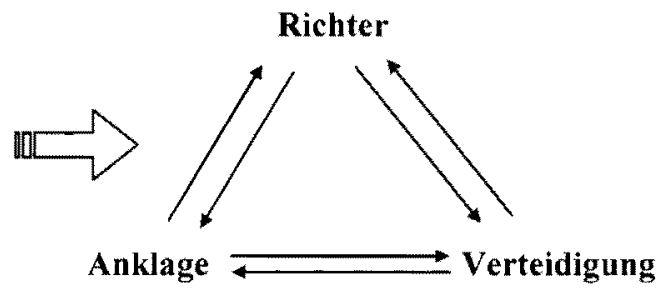

Abb. 4: Kommunikationskanäle in der ursprünglichen und gerichtlichen Konfliktkommunikation

Mit der Triangulation der Rechtskommunikation werden insofern unsichere, aber entscheidungsrelevante Loyalitäten in das Konfliktsystem eingeführt, die den Prozess der Entscheidungsfindung unsicher werden lassen:45 Man glaubt sich im Recht, kann sich aber nicht sicher sein, letzten Endes Recht zu bekommen. Konstitutiv dafür ist die Position

45 "[D]as Auftreten des Dritten bedeutet Übergang, Versöhnung, Verlassen des absoluten Gegensatzes - freilich gelegentlich auch die Stiftung eines solchen", so schon SMMEL, Soziologie (Fn. 44), 75 
eines Richters. Die Funktion seines Standpunkts entbindet ihn von den Loyalitätspflichten hinsichtlich einer spezifischen Konfliktposition und macht sein Entscheidungsverhalten sozial kontingent. ${ }^{46}$ Demgemäß steht ihm ein distanzierter Beobachtungsposten $z u$, von dem aus er die Prätentionen der Parteien (bzw. deren Stellvertreter) beobachten und handlungsentlastet beurteilen kann. Weniger offensichtlich ist, dass den Parteien (bzw. ihren Stellvertretern) ihrerseits ähnliche Beobachtungsmöglichkeiten zur Verfügung stehen, an denen sich gegebenenfalls schon ablesen lässt, in welchen Hinsichten der Richter die eigene Version anerkennen oder ablehnen wird. Die Anzeichen richterlichen Koalierens (bzw. Defektierens) mit der eigenen (oder fremden) Konfliktposition werden auf Grund der Interaktionsförmigkeit des Verfahrens und entsprechender Signalwirkungen von den Anwesenden zumeist genau registriert. ${ }^{47}$ Aus konflikttheoretischer Sicht liegt in den wechselseitigen Beobachtungsmöglichkeiten ein Schlüssel zum Verständnis von Zermürbungsprozessen des gerichtlichen Konfliktmanagements, das die Konfliktbeteiligten (bzw. deren Interessenvertreter) mithin zum Einlenken, Nachgeben bzw. zur Abänderung einer Prozessstrategie veranlassen kann. Die zumindest teilweise Sichtbarkeit kontingenter Konfliktallianzen macht den Prozess des Entscheidens für alle Anwesenden indes zu einer empirisch greifbaren Realität, an der sich ablesen lässt, wie sich die eigenen und fremden Darstellungen vor Gericht entscheidungswirksam gestalten. In diesem Sinne ist die Beobachtbarkeit des Entscheidungsvorgangs ebenfalls ein wesentlicher Aspekt der Schwächung konfliktstabiler

\footnotetext{
${ }^{46}$ Entsprechend spielt die Darstellung richterlicher Unparteilichkeit in der Gerichtskommunikation eine nicht unbedeutende Rolle. Sie folgt aus dem Grundsatz, dass sich ein Richter bis zum Urteilsspruch allen direkten Äußerungen über die Glaubwürdigkeit von Zeugenaussagen enthält, vgl. etwa J. MAXWELL ATKINSON, Displaying Neutrality: Formal Aspects of Informal Court Proceedings, in: PAUL DREW/JOHN HerITAGE (Hrsg.), Talk at Work: Interaction in Institutional Settings. Cambridge 1992, 199. Das gilt in vergleichbarer Weise auch für verschiedene andere soziale Settings, in denen Neutralität zum professionellen Rollenbild dazugehört, vgl. beispielsweise für Interviewsituationen STEVEN E. CLAYMAN, Displaying Neutrality in Television Interviews, in: Social Problems 35 (1988), 474; DERS., Footing in the Achivement of Neutrality: The Case of News Interview Discourse, in: PAUL DREW/JOHN HERITAGE (Hrsg.), Talk at Work: Interaction in Institutional Settings. Cambridge 1992, 137.

47 Beispielsweise lässt sich die Anerkennung der Glaubwürdigkeit von Sachverhalten vor Gericht an verschiedenen Äußerungsformen des Richters indirekt erschließen, so etwa an Bezweiflungspartikeln, Ironie, Normalitätsunterstellungen, Vorhalten und Ährlichem mehr, vgl. STEPHAN WOLFF/HERMANN MÜlLER, Kompetente Skepsis. Eine konversationsanalytische Untersuchung zur Glaubwürdigkeit in Strafsachen. Opladen 1997, mit aufschlussreichen Kommunikationsdaten; speziell zu Normalitätsunterstellungen vgl. auch LUDGER HOFFMANN, Rechtsdiskurse zwischen Normalität und Normativität, in Ulrike Haß-ZumkeHR (Hrsg.), Sprache und Recht. Berlin 2002, $80 \mathrm{ff}$.
} 
Beziehungsstrukturen. Dieser Zusammenhang wird durch die nachfolgende Abbildung illustriert:

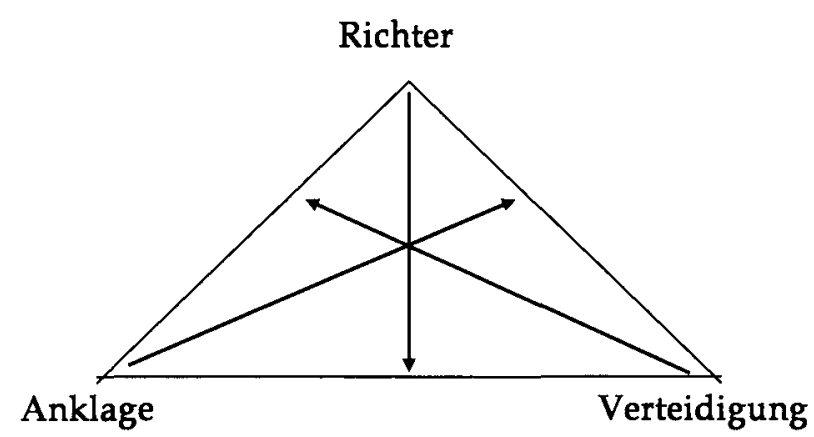

Abb. 5: Beobachtungsmöglichkeiten in der triangulierten Gerichtskommunikation

Gerichtsverfahren bauen auf eine Rollendifferenzierung auf, die sicherstellt, dass die Betroffenen selbst nicht entscheiden, jedoch miterleben, wie sich der Entscheidungsvorgang kommunikativ realisiert. Dazu werden die Kommunikationsmöglichkeiten der Entscheidungsbetroffenen stark limitiert, während die der Professionellen stark expandieren. Aus konflikttheoretischer Sicht besteht die eigentliche Leistung dieses Vorgangs in der kommunikativen Durchbrechung einer interpunktierten Konfliktrealität, die für die wechselseitig kontinuierliche Schuldzuschreibung in Beziehungskonflikten wesentlich ist und dort die Bedingungen ihrer Fortführbarkeit aus dem Konfliktprozess heraus selbstbezüglich begründet. Interpunktierte Konfliktansichten sind zwar auch vor Gericht keine Seltenheit, jedoch sind ihnen hinsichtlich ihrer Folgen klar sichtbare Grenzen gezogen. Beispielsweise wird das individuelle Konflikterleben schon auf dem Wege der Klärung von Tatsachenfragen nachhaltig unterminiert und damit die Sachverhaltsdarstellung von subjektiv eigenwilligen Kausalunterstellungen entkoppelt. Die Frage der Schuld wird in Feststellungen über Verhalten prozessiert, das interpunktierte Konflikterleben in seine Detailkomponenten auflöst und dadurch zerstreut. Die für den Beziehungskonflikt typische ,Ich/ Du-Distinktion' wird damit in die für Sachkonflikte kennzeichnende Ja/Nein-Distinktion' zurückübersetzt, der Konfliktvorgang in seiner Darstellung dadurch versachlicht. ${ }^{48}$ In den Begrifflichkeiten der An-

48 Dies hat im Übrigen auch schon SIMMEL, Soziologie (Fn. 44), 77, ähnlich deutlich erkannt. Die Einführung eines Dritten in die dyadische Beziehung bewirke, dass „der für 
schuldigungskommunikation wird zuerst die Frage nach der antecedent action bzw. nach dem actor-agent gestellt, bevor Motive und Absichten zugerechnet werden, was im Übrigen ausschließlich dem Gericht vorbehalten ist.

Schließlich wird das interpunktierte Konflikterleben systematisch auch durch das gerichtstypische turn-taking unterlaufen. Das turn-taking bezeichnet in der Konversationsanalyse regelhafte Strukturmerkmale der Redezugorganisation, die sich settingspezifisch meist signifikant unterscheiden. Demnach ist es keineswegs eine Frage des Beliebens, wer wann wie zu einem anderen spricht. Bei der ärztlichen Visite beispielsweise ist es meist der Arzt, der Fragen stellt, während der Patient die Antworten gibt. Wissenschaftliche Vorträge wiederum zeichnen sich durch ein ganz anderes turn-taking aus, sofern dem Vortragenden für längere Zeit ein Rederecht eingeräumt wird, während man selbst die eigenen Mitteilungsbedürfnisse bis auf weiteres unterdrückt. Korrespondierend zu den Absichten der jeweiligen Situation sieht das turn-taking also spezielle Austauschbeziehungen für turn-Übergaben vor, es etabliert Regeln der Sprecherwahl und konditioniert die Anschlussoptionen der jeweiligen Rede. ${ }^{49}$ Im Hinblick darauf sind Sinn und Funktion einer kommunikativen Äußerung eng mit den formalen Regeln ihrer Herstellung verknüpft. Die regelgeleitete Sequenzialität des turn-taking systems ist insoweit indexikalisch, als sie auf formale Eigenschaften des sozialen Settings zurückverweist und über seine Ordnungs- und Struktureigenschaften Aufschluss vermittelt.

Dieser Zusammenhang gilt für die Gerichtskommunikation entsprechend. Wie die Untersuchungen hierzu zeigen, ist das turn-taking in den Verfahren der Gerichtsbarkeit außerordentlich stark restringiert. ${ }^{50}$

alle Verständigung verhängnisvolle Zirkel vermieden wird: daß die Heftigkeit des einen die des anderen hervorruft, diese letztere Tatsache aber zurückwirkend die Heftigkeit des ersteren wieder steigert, und so fort, bis es keinen Halt mehr gibt. Dazu kommt, daß jede Partei nicht nur Objektiveres hört, sondern sich auch objektiver äuBern muß, als bei unmittelbarem Gegenüberstehen. Denn es muß ihr jetzt darauf ankommen, auch den Vermittler für ihren Standpunkt zu gewinnen."

${ }^{49}$ Grundlegend: Harvey SaCKS/Emanuel A. SChegloff/Gail Jefferson, A Simplest Systematics for the Organization of Turn-Taking for Conversation, in: Language 50 (1974), 696; EMANUEL A. SCHEGLOFF, Sequencing in Conversational Openings, in American Anthropologist 70 (1968), 1075.

50 Bahnbrechend: J. MAXWELL ATKINSON/PAUL DREW, Order in Court: The Organization of Verbal Interaction in Judicial Settings. Atlantic Highlands 1979; ROBERT DUSTAN, Context for Coercion: Analyzing Properties of Courtroom 'Questions', in: British Journal of Law and Society 7 (1980), 61; ROBERT M. HAYDEN, Turn-Taking, Overlap, and the Task at Hand: Ordering Speaking Turns in Legal Settings, in: American Ethnologist 14 (1987), 251; BRENDA DANET, An Overview of 15 Years of Research, in: HOWARD Giles/WALTER P. ROBinson (Hrsg.), Handbook of Language and Social Psychology. Chichester 1990, 537. 
Während die Redezugorganisation im Konflikt mit wachsender Konfliktintensität zunehmend weniger geordnet verläuft, d. h. die Konfliktbeteiligten sich in ihren Mitteilungsmöglichkeiten zunehmend häufiger und rigider beschneiden,51 wird vor Gericht gerade dies mit größter Sorgfalt vermieden. Das gerichtliche turn-taking arbeitet den interaktiven Prozessen der Konflikteskalation insofern deutlich entgegen, indem es formal diejenigen Strukturen der Redezugorganisation unterstützt, die eine Verständigung eher erleichtern. Vor allem aber wird das gerichtliche turn-taking strukturell vom Frage/AntwortFormat ${ }^{52}$ dominiert, was dem Verfahren minutiöse Kontrollmöglichkeiten über die kommunikativen Ablaufprozesse ermöglicht. Wer fragt, engt den Horizont möglicher Antwortinhalte ein und definiert damit die Themen der Sinnproduktion, von denen der andere nicht unbegründet abweichen kann, ohne damit Misstrauen, Nachfragen oder Unmut zu wecken. Ebenso schreibt die Frage ihren second turn-part, also Antworten vor, was alternative Reaktions- oder Mitteilungsformen strukturell dispräferiert. Wer Fragen ausweicht oder unbeantwortet lässt, macht sich damit - nicht nur vor Gericht - verdächtig. ${ }^{53}$ Darüber hinaus ist die Zuteilung von Fragerechten vor Gericht (wie in anderen sozialen Settings übrigens auch) typischerweise asymmetrisch organisiert. Sofern das Fragerecht strukturell Macht indiziert, steht es fast ausschließlich nur den Mitgliedern des Gerichts zur Verfügung.

Aus konflikttheoretischer Sicht fällt weiterhin auf, dass das Frage/ Antwort-Format im gerichtlichen turn-taking der Reproduktion sozialer Konflikte die elementaren Ressourcen entzieht. Zumindest fällt es schwerer, diese innerhalb von Frage und Antwort zu prozessieren. Dies gilt bereits für die den Sachkonflikt begründende Widerspruchskommunikation. Denn bezüglich einer Frage fungiert das ,Nein' - anders als in der Widerspruchskommunikation - nicht mehr länger als Ablehnung einer Sinnzumutung, sondern primär als Antwort auf die vorausgehende Frage. Während die Sinnzumutung typischerweise auf Zustimmung spekuliert (Der Wagen kam von links), hält die Frage die

51 Vgl. MESSMER, Der soziale Konflikt (Fn. 20), 171 ff. mit weiterführenden Analysen.

52 Zum Frage-Format vgl. näher LINDSEY CHURCHILL, Questioning Strategies in Sociolinguistics. Rowley (Mass.) 1978; ESTHER N. GOODY (Hrsg.), Questions and Politeness: Strategies in Social Interaction. Cambridge 1978; DUSTAN, Context for Coercion (Fn. 50); SANDRA HARRIS, Questions as a Mode of Control in Magistrates' Courts, in: International Journal of Sociology of Language 49 (1984), 5.

${ }^{53}$ Vgl. näher HARVEY SACKS, The Inference-Making Machine: Notes on Observability, in: TEUN A. VAN DIJK (Hrsg.), Handbook of Discourse Analysis. Vol. 3: Discourse and Dialogue London 1985, 13, mit dem Hinweis auf Angemessenheit, Schlüssigkeit und Vollständigkeit als Bedingungen einer glaubhaften Antwort; vgl. ferner SANDRA HARRIS, Evasive Action: How Politicians Respond to Questions on Political Interviews, in: PADDY SCANNELL (Hrsg.), Broadcast Talk. London 1991, 76. 
Alternative zwischen Zustimmung und Ablehnung strukturell in der Schwebe (Kam der Wagen von links?). Im einen Fall ist Widerspruch, im anderen eine Antwort die erwartete Reaktion. Vergleichbares gilt für die Anschuldigungskommunikation des Beziehungskonflikts. Das attributive , $\mathrm{Du}^{\prime}$ der Anschuldigungskommunikation ist im Rahmen des Frage/Antwort-Formats nur noch gebrochen mitteilbar, und zwar aus denselben Gründen, wie schon bei der Widerspruchskommunikation, sofern es auch hier die gegenteilige Alternative einräumen muss. Die Verantwortungszurechnung stellt fest, die Frage dagegen hält die Feststellung in der Schwebe. Dasselbe gilt schließlich auch für den auf Drohkommunikationen aufbauenden Machtkonflikt. Drohkommunikationen sind vor Gericht ohnehin ausgeschlossen und werden sofort sanktioniert, es sei denn, sie werden vom Richter geäußert. Pragmatisch laufen sie den Absichten des Frage/Antwort-Formats im Grundsatz zuwider, sofern die Drohung auf die Eliminierung von Reaktionsalternativen, die Frage hingegen auf Offenheit zielt.

Wir halten fest: Die Umformung sozialer Konflikte in der Sprache des Rechts deformiert die Strukturen der Konfliktreproduktion beträchtlich. Sie unterbricht die Unmittelbarkeit und Reaktivität sozialer Beziehungsmuster in der Konfliktinteraktion, etabliert alternative Relevanzgesichtspunkte des Durchsetzens und Entscheidens und zwingt die Rechtsbetroffenen zur grundlegenden Umorientierung ihrer Konfliktstrategien. Die binär strukturierte Konfliktbeziehung wird in gerichtseigene Kommunikationskanäle umdirigiert und damit der Kontrolle durch die Parteien entzogen. Mit zunehmender Verfahrenskomplexität sehen sich die Entscheidungsbetroffenen zum passiven Nachvollzug fremder Entscheidungsprozesse verurteilt. Diese Form des Konfliktmanagements hat zudem den Effekt, dass das interpunktierte Konflikterleben der Parteien strukturell desorientiert, gewissermaßen von innen heraus ausgehöhlt wird. Darüber hinaus wird die Reproduktionsfähigkeit regenerativer Konfliktzirkel auf Grund von Tatsachenfeststellungen weitestgehend wieder, geerdet'. Unterstützt durch das gerichtliche turn-taking, das hauptsächlich im Medium von Frage und Antwort operiert, werden die zirkulären Muster des interpunktierten Konflikterlebens gewissermaßen in ihre Einzelbestandteile zerlegt und der Interpunktionsmechanismus selbst neutralisiert. Widerspruch, Anschuldigung oder Drohung sind nur noch insoweit mitteilbar, wie es gelingt, sie in das Format einer Antwort zu kleiden. 


\section{Fallbeispiel einer gerichtlichen Zeugenvernehmung}

Anhand einer kurzen Textsequenz ${ }^{54}$ aus der gerichtlichen Zeugenvernehmung wollen wir die bisherigen Überlegungen an einem Fallbeispiel illustrieren. Diesem liegt der Sachverhalt eines Vergewaltigungsvorwurfs zugrunde, der insofern strittig ist, als beide Seiten ihr Verhalten gemäß dem Muster einer interpunktierten Konfliktrealität unterschiedlich interpretieren. Täter (bzw. Beklagter) und Opfer (bzw. Klägerin oder Zeugin) waren zum Zeitpunkt des Vorfalls einander flüchtig bekannt. An diesem Abend trafen sie in einer Gaststätte zusammen, wo es zu einer beiläufigen Unterhaltung kam. Später begleitete der Beschuldigte die Zeugin hinaus und fragte, ob er sie zum Abschied küssen dürfte. Anschließend lud er sie zu einem Hamburger bei McDonalds in einer nahe gelegenen Shoppingmall ein. Auf dem Weg dorthin bog er in einen abseits gelegenen Seitenweg ab, wo es dann später zu dem fraglichen Vorfall kam.

Obwohl der faktische Ereignisablauf dieses Abends weitgehend unstrittig ist, bestehen zwischen den Sachverhaltsdarstellungen beider Seiten dennoch grundlegende Differenzen. Aus Sicht der Zeugin handelte es sich bei diesem Vorfall um den Tatbestand einer Vergewaltigung, da es gegen ihren Willen zum Geschlechtsverkehr mit dem Beschuldigten kam. Dass der Beklagte erkennbar gegen den Willen der Betroffenen handelte, ist aus seiner Sicht (bzw. aus der der Verteidigung) jedoch fraglich. Vielmehr habe er das ,Nachgeben' der Klägerin in der Gaststätte wie auch später als Aufforderung für weitere Annäherung aufgefasst, so dass ihm sein Verhalten situativ gerechtfertigt scheint. Soweit die zitierten Textbeispiele dies zu erkennen geben, beruht der fragliche Vorfall auf einer gegenläufig interpunktierten Verkettung von Ursachen und deren Folgen: Demnach ,handelte' der Beschuldigte vornehmlich deswegen, weil die Klägerin sich nachgiebig zeigte (sie ließ sich auf ein Gespräch ein, ließ sich hinausbegleiten und zum Abschied küssen und nahm schließlich noch eine Einladung zum Restaurantbesuch an). Aus Sicht der Zeugin verhält es sich demgegenüber aber so, dass sie sich deswegen nachgiebig zeigte, weil der Beklagte sie immer weiter bedrängte. Die Kausalstruktur der Ereignisabfolge innerhalb dieser Konfliktbeziehung wird also unter dem Gesichtspunkt von Sich-Nähern und Nachgeben interpunktiert: Ein Verhalten (Sich-

\footnotetext{
${ }^{54}$ Entnommen aus PAUL DREW, Contested Evidence in Courtroom Cross-Examination: The Case of a Trial for Rape, in: DREW/HERITAGE, Talk at Work (Fn. 46), 470, $478 \mathrm{ff}$. vgl. auch DERS., Strategies in the Contest Between Lawyer and Witness in CrossExamination, in: JUdITH N. LeVI/ANNE GRAFFAM WALKER (Hrsg.), Language in the Legal Process. New York 1990, 39, 45 ff.
} 
Nähern) ist in einem Fall Ursache für eine spezifische Reaktion (Nachgeben), im anderen Fall hingegen Reaktion auf eine spezifische Ursache.

Im Zentrum strittiger Schuldfragen steht insofern auch hier die Frage nach der Kausalität, wer ursächlich handelt und wer auf das Handeln des anderen bloß reagiert. Entsprechend rechnen sich beide Seiten wechselseitig die Verantwortung für den unerfreulichen Sachverhalt $\mathrm{zu}$, um das je eigene Verhalten als legitim zu begründen. Die beiden nachfolgenden Textsequenzen geben hierzu einen Ausschnitt aus dem gerichtlichen Kreuzverhör wieder, bei dem der Verteidiger den Nachweis $z \mathfrak{u}$ führen versucht, dass die Klägerin, die hier als Zeugin aussagt, durch ihr Verhalten zu dem Tathergang kausal ursächlich beitrug und insofern mitverantwortlich ${ }^{55}$ an dem fraglichen Vorfall war:

[1]

01 A: An' you went to a $\underline{\text { : }}$ uh $(0.9)$ uh you went to

02 a ba:r? in ((city)) (0.6) is that correct?

$03(1.0)$

04 W: Its a clu:b.

[2]

01 A: Its where uh (.) gi:rls and fella:s

02 meet isn't it?

$03 \quad(0.9)$

04 W: People go: there.

An den Reaktionen der Zeugin wird soviel klar, dass sie die Fragen der Verteidigung nicht als Fragen, sondern vornehmlich als Anschuldigung ,hört' und sich ferner darüber bewusst ist, welche schuldrelevanten Inferenzen sich damit verknüpfen. Beispielsweise legt die Ortsbezeichnung (ba:r) Schlussfolgerungen auf mögliche Kontaktabsichten mit dem Tatverdächtigen nahe. Die Kennzeichnung des Ortes steht insofern indexikalisch für ein spezielles Verhaltensmotiv, aus dem sich mögliche Anhaltspunkte für die Mitschuld der Zeugin ergeben: Wer eine Bar aufsucht, sucht häufig auch den (zwischengeschlechtlichen) Kontakt zu Fremden. Ein solches Verhaltensmotiv wiederum lässt

\footnotetext{
${ }^{55} \mathrm{Zu}$ den Techniken der Aufdeckung unglaubhafter Zeugenaussagen im Zusammenhang sexueller Gewaltdelikte, insbesondere zur Typisierung konventioneller Vergewaltigungs-Szenarien vgl. LISA FROHMANN, Discrediting Victims' Allegations of Sexual Assault: Prosecutoral Accounts of Case Rejections, in: Social Problems 38 (1991), 213, 217. Frohmann spricht in diesem Zusammenhang auch von ulterior motives: „Ulterior motives rest on the assumption that a woman consented to sexual activity and for some reason needed to deny it afterwards", ebd., 221.
} 
Rückschlüsse auf den moralischen Charakter der Betroffenen zu und informiert über ihre Risikobereitschaft im Umgang mit Fremden. In diesem Sinne greift die Zeugin der logischen Verkettung schuld-implikativer Inferenzmöglichkeiten vor: Eine die Person potentiell negativ charakterisierende Kennzeichnung des Ortes wird durch eine neutralere Ortsbezeichnung ersetzt ( $\underline{\text { clu:b}}$ ). Dieselbe Präventivtaktik greift auch in Textfragment [2]. Wiederum wird in der Rekonstruktion der Vorgeschichte des Tathergangs ein durch die Verteidigung konnotiertes Motiv offenkundig (where uh (.) gi:rls and fella:s meet), das möglicherweise Rückschlüsse auf schuldrelevante Verhaltensabsichten der Zeugin erlaubt. Erneut greift die Zeugin der Anschuldigung vor: Der Suggestion einer speziellen, für sie verfänglichen Verhaltensabsicht wird durch Angabe eines allgemeineren - und deshalb auch: sozial konsensfähigeren - Verhaltensmotivs entgegengetreten. Bei diesem Club handelt es sich um einen Ort, den alle frequentieren, nicht nur Leute mit einem speziellen Motiv.

Kaum merkliche Begriffsvariationen innerhalb einer kausallogischen Verkettung der Anschuldigungskommunikation haben mitunter weit reichende Folgen. Die örtliche Präsenz lässt auf spezifische Handlungsabsichten schließen, und diese wiederum geben Auskunft über die moralische Integrität der Person. Dieser Kausalkonnex erklärt im vorliegenden Fall die hohe Wort- und Begriffssensibilität des betroffenen Opfers. Deutlich wird, dass schon die Andeutung der Möglichkeit einer Verantwortungszuschreibung ausreicht, um entsprechende Defensivreaktionen zu generieren. Dies ist insofern verständlich, da jede Mitverantwortung am Sachverhalt Schuld impliziert, die bestehende Wiedergutmachungsansprüche bzw. Tatausgleiche unter Umständen hinfällig macht.

Ferner fällt auf, dass es sich bei den zitierten ,Fragen' des Verteidigers mehr um Sachverhaltsfeststellungen handelt, jeweils angereichert mit einer Zustimmungserwartung in Frageintonation. Die ,Fragen' sind jeweils so angelegt, dass sie auf die Bestätigung des frageeinleitenden Statements zielen (is that correct?/isn't it?). Eine Möglichkeit zur Ablehnung ist formal zwar gegeben, rhetorisch aber dispräferiert. Im vorliegenden Fall wird der Sachverhalt an sich auch nicht verneint, die schuldimplikativen Sinnkomponenten (ba:r/gir:ls an fella:s) jedoch einer Korrektur unterzogen. Damit bestätigt die Zeugin den Sachverhalt, gibt ihm jedoch eine für sie unverfänglichere Wendung. Aufgrund des Frageformats kann sie der Darstellung des Verteidigers jedoch keine eigene Version entgegenhalten, sondern allenfalls präventiv auf dessen Unterstellungen reagieren, und das heißt hauptsächlich: schuldimplikative Inferenzen blockieren. Die Möglichkeit zur Darstellung ihrer eigenen 
Sachverhaltsversion muss demgegenüber zwangsläufig unberücksichtigt bleiben, sie ist im Zuge des Frage/Antwort-Formats praktisch kaum ausführbar. Damit wird die Zeugin zwangsläufig zur Komplizin bzw. Ko-Produzentin einer Konfliktversion, die ihren Interessen zuwiderläuft und deren vornehmliche Absicht darin besteht, das Gericht von ihrer Mitschuld zu überzeugen. Dies zeigt auch ein Ausschnitt aus dem Schlussplädoyer der Verteidigung: 56

[3]

01 A: Now it seems to me (a) situation that uhr:

02 (0.5) that uhr, (0.7)

03 uhr::, whe:re yuh have two $(0.2)$

04 young people (0.6) who (0.6) appea:r (0.4)from their

05 testimony to be interested in each other (...)

06 he kissed her goodnight, (0.5)

07 an' I reckon she wannah, $(0.8)$

08 no question about that $(0.3)$

09 a'right? here's two people who ar:e er interested in

10 each other.

\section{Konflikt, Recht, Gesellschaft}

Die Sprache des Rechts und die des Konflikts weisen in Grundzügen isomorphe Struktureigenschaften auf, in dem Sinne, dass im Mittelpunkt der sozialen Beziehung eine Unvereinbarkeit existiert, die sich kommunikativ als Anschuldigung mitteilt. Für den Beziehungs- wie für den Rechtskonflikt gilt, dass die Frage nach der Verantwortlichkeit für ein inkriminiertes Verhalten strittig ist. In dieser Form sind Konflikte vor Gericht nicht nur zugelassen und anerkannt, sondern sie werden im Hinblick auf die Einseitigkeit ihrer Interessensverfolgung zudem pointiert auf die Spitze getrieben. Die "materialrechtliche verwertbare Wahrheit" muss mit der "unformalisierten wirklichen Wahrheit" (Naucke) nicht unbedingt harmonieren. Sie muss jedoch mit rechtseigenen Mitteln darstellbar sein, um vor Gericht Anerkennung zu finden..$^{57}$ Kein Mittel ist zu gering, kein Hinweis zu unbedeutend, wenn es

\footnotetext{
56 DREW, Contested Evidence in Courtroom Cross-Examination (Fn. 54), 518, Fn. 7.

57 In der Prozesspause eines von $\mathrm{mir}$ beobachteten Verfahrens äußerte sich ein Verteidiger gegenüber einer Zeugin der Klägerpartei, die sich über die unwahre Darstellung des Prozessgegners mokierte, folgendermaßen: „Was regen Sie sich so auf? Was erwar-
} 
gilt, die Glaubwürdigkeit der eigenen Sachverhaltsdarstellung zu unterstreichen. Diese Ausschöpfung sämtlich verfügbarer Prozessmittel ist nur zu verständlich, handelt es sich doch um Entscheidungsvorgänge im Rahmen einer binär strukturierten Recht/Unrecht-Disjunktion, die den Konflikt formal oft unwiderruflich zu Gunsten des einen und zu Ungunsten des anderen beenden.

Gegenüber diesen Zuspitzungsmöglichkeiten wartet das Recht im Gegenzug mit Limitierungen auf, welche die Negationspotentiale des Konflikts in spezifischer Weise untergraben. So werden die Streitinhalte in die Form eines Rechtsgegenstands überführt und die Konfliktthemen nach Maßgabe rechtlicher Normen weiter entschieden. Ausschlaggebend dafür sind nicht länger individuelle Stärke oder das Durchhaltevermögen im Konflikt, sondern Sachverhaltsfeststellung, Glaubwürdigkeitsprüfung und Zeugenbeweis. Es reicht nicht mehr aus, sich in seinen Standpunkten unnachgiebig zu zeigen, wenn nicht auch das Gericht von ihrer Richtigkeit überzeugt werden kann. Die kognitive Rigidität und Monovalenz stereotyp verfestigter Konfliktwahrnehmungen sieht sich daher vielfältigen $Z$ weifeln ausgesetzt, denen besonders die subjektiv eingefärbten Konfliktkomponenten zum Opfer fallen. Die Hitze einer persönlich-unmittelbaren Konfliktkonfrontation wird damit gewissermaßen, heruntergedimmt'. Zugleich wird dem Ursprungskonflikt ein rechtseigenes Kommunikationssystem übergestülpt, in dem die Konfliktbetroffenen nur noch bedingt berücksichtigt werden.

Die Charakterisierung von Gerichtsverfahren als einem konfliktbezogenen Entscheidungsvorgang greift daher entschieden zu kurz. Das Verfahren selbst ist immer auch praktiziertes Konfliktmanagement, das auf die Aufhebung selbstrekursiver Konfliktzirkel zielt. Allein durch Tatsachenfeststellungen und Normbezug werden die persönlichen Überzeugungen der Konfliktbetroffenen kommunikationsstrukturell wirksam und nachhaltig neutralisiert. Damit entsteht eine eigentümliche Distanz zwischen dem Konflikt und den Bedingungen seiner Lösung. Die Konfliktbetroffenen werden zunehmend voneinander separiert, konflikttypische Beziehungsmuster werden entzerrt und zahlreiche für die Konfliktreproduktion maßgebliche Äußerungsformen unterbunden. Damit blockiert das Gericht letzthin auch die kognitiven und sozialen Dynamiken der Konflikteskalation. Freilich ist damit der Konflikt weder gelöst noch beendet, im rechtlichen Sinne aber entschieden und sozial reguliert.

ten Sie hier? Die Wahrheit? Wir sind hier vor Gericht. Nirgendwo sonst wird soviel gelogen wie vor Gericht: 
Von einem gesellschaftstheoretischen Standpunkt aus gesehen lässt sich zeigen, dass die Ausdifferenzierung sozialer Konflikte überall dort auf Widerstand stößt, wo auf Grund umgreifender sozialer Dissoziierung und gewaltförmiger Durchsetzungsstrategien basale Mechanismen der Sozialintegration in Frage gestellt, bedroht oder ausgehöhlt werden. Den universell verfügbaren Negationspotentialen des Konflikts stehen ebenso gesellschaftlich mächtige Institutionen der Konfliktdämpfung gegenüber, deren Funktion speziell in der Abfederung und Vermeidung sozial destruktiver bzw. pathologischer Konfliktfolgen liegt. Die kommunikative Machtausübung und Kontrolle in den Verfahren des Rechts verhält sich proportional zur Explosivität sozialer Konflikte. Sie ist Teil und Ergebnis eines gesellschaftlichen Konfliktmanagements, das den Anspruch erhebt, alle relevanten Unvereinbarkeiten mit rechtseigenen Mitteln auf friedlichem Weg zu entscheiden.

Innerhalb der Gesellschaft stoßen wir zudem auf elementare, an Kooperation, Vertrauen und Affirmation angelehnte Erwartungsstrukturen, die das Sozialverhalten normativ und regelhaft orientieren. Generell gilt, dass die Normen des Takts, der Höflichkeit und der Achtung einer umfassenden Konfliktrepression dienen, die man typischerweise nur dann übertritt, wenn man bereit ist, den Konflikt zu vertiefen. Symbolisch generalisierte Kommunikationsmedien befinden darüber hinaus, was an Kommunikationen zulässigerweise bejaht oder verneint werden kann und regulieren über weite Bereiche das gesellschaftliche Negationspotential. Das gilt vor allem für die Kommunikationsmedien ,Recht' und ,Macht', aber auch für ,Wahrheit', ,Geld' oder ,Liebe' ${ }^{58}$ Die elementaren Normen der Interaktion bilden ebenso wie die symbolisch generalisierten Kommunikationsmedien und die institutionell ausdifferenzierten Verfahren des Rechts zusammen eine wirksame Barriere hinsichtlich des sozialen Konfliktrisikos.

Betrachtet man die Gesellschaft als eine Art Fernumwelt des Konflikts, so wird deutlich, dass diese ihre Sozialstrukturen nicht uneingeschränkt dem Konflikt aussetzen darf, will sie damit ihren eigenen Weiterbestand nicht gefährden. Andererseits aber, und dieser Aspekt wiegt nicht weniger schwer, sind Konflikte gesellschaftlich nicht ausschließlich nur dysfunktional, pathologisch oder destruktiv: Insoweit der Widerspruch auf Inkongruenzen und Unvereinbarkeiten der gesellschaftlichen Sinnverarbeitung aufmerksam macht, lässt sich sein Beitrag zur Korrektur, Veränderung oder zur Verbesserung sozialstruktureller Einrichtungen kaum überschätzen. Dieser Sachzusammenhang

${ }^{58}$ Vgl. NiKLAS LuHMANN, Einführende Bemerkungen zu einer Theorie symbolisch generalisierter Kommunikationsmedien, in: DERS., Soziologische Aufklärung 2: Aufsätze zur Theorie der Gesellschaft. Opladen 1975, $170 \mathrm{ff}$. 
gilt für Interaktionen ebenso wie für Gruppen und Organisationen, 59 aber auch für Nationen. ${ }^{60}$ Ohne Konfliktrisiko könnte man nicht widersprechen, und weder gäbe es Abgrenzung noch die Möglichkeit zur Entwicklung einer eigenständigen Identität. Ein soziales System, gleich welcher Art, das den Konflikt als Prämisse seines Weiterbestands ignorierte, könnte weder seine Prozesse noch seine Strukturen entwicklungs- und umweltadäquat variieren. Es wäre vielmehr zur Stagnation und Starrheit verurteilt und hätte kaum Chancen zum Überleben. Soziale Systeme benötigen den Konflikt, um - wie Luhmann sagt ${ }^{61}$ - sich rechtzeitig gegen innere und äußere Bestandsgefährdungen zu immunisieren. Deshalb kann der Gesellschaft die das Soziale einerseits ermöglichende, andererseits zersetzende Kraft des Konflikts auch nicht gleichgültig sein. Gesellschaft benötigt Konflikt. Seinen destruktiven Folgen müssen jedoch Grenzen gesetzt werden, insbesondere im Hinblick auf die Möglichkeit gewaltsamer Durchsetzungsstrategien. All dies spiegelt sich zuletzt auch in den Kommunikationen des Rechts.

${ }^{59} \mathrm{Vgl}$. GERD NOLLMANN, Konflikte in Interaktion, Gruppe und Organisation - Zur Konfliktsoziologie der modernen Gesellschaft. Opladen 1997.

${ }^{60}$ Vgl. etwa MORTON DEUTSCH, Preventing World War III: A Psychological Perspective, in: Ders., Distributive Justice: A Social-Psychological Perspective. New Haven (Conn.) $1985,262 \mathrm{ff}$.

${ }^{61}$ Vgl. NikLas Luhmann, Konflikt und Recht, in: DeRs., Ausdifferenzierung des Rechts: Beiträge zur Rechtssoziologie und Rechtstheorie. Frankfurt am Main 1981, 92, 97; DERS., Soziale Systeme (Fn. 21), $504 \mathrm{ff}$. 